\title{
The Dynamics of Epidemic Model with Two Types of Infectious Diseases and Vertical Transmission
}

\author{
Raid Kamel Naji and Reem Mudar Hussien \\ Department of Mathematics, College of Science, University of Baghdad, Baghdad, Iraq \\ Correspondence should be addressed to Reem Mudar Hussien; reem.m.hussien@gmail.com
}

Received 27 August 2015; Accepted 10 December 2015

Academic Editor: Zhen Jin

Copyright ( 2016 R. K. Naji and R. M. Hussien. This is an open access article distributed under the Creative Commons Attribution License, which permits unrestricted use, distribution, and reproduction in any medium, provided the original work is properly cited.

An epidemic model that describes the dynamics of the spread of infectious diseases is proposed. Two different types of infectious diseases that spread through both horizontal and vertical transmission in the host population are considered. The basic reproduction number $R_{0}$ is determined. The local and the global stability of all possible equilibrium points are achieved. The local bifurcation analysis and Hopf bifurcation analysis for the four-dimensional epidemic model are studied. Numerical simulations are used to confirm our obtained analytical results.

\section{Introduction}

Mathematical models can be defined as a method of emulating real life situations with mathematical equations to expect their future behavior. In epidemiology, mathematical models play role as a tool in analyzing the spread and control of infectious diseases. Although one of the most famous principles of ecology is the competitive exclusion principle that stipulates "two species competing for the same resources cannot coexist indefinitely with the same ecological niche" $[1$, 2], Volterra was the first scientist who used the mathematical modeling and showed that the indefinite coexistence of two or more species limited by the same resource is impossible [3]. Moreover, Ackleh and Allen [4] were the first who used the competitive exclusion principle of the infectious disease with different levels in single host population.

It is well known that one of the most useful parameters concerning infectious diseases is called basic reproduction number. It can be specific to each strain of an epidemic model. In fact the basic reproduction number of the model is defined as the maximum reproduction numbers of other strains [57]. Diekmann et al. [8] had studied epidemic models with one strain, while Martcheva in [9] studied the SIS-type of disease with multistrain. However, Ackleh and Allen [10] studied $S I R$-type of disease with $\mathbf{n}$ strain and vertical transmission.

Keeping the above in view, in our proposed model two strains with two different types of infectious diseases are considered. Accordingly two different reproduction numbers are obtained and then competitive exclusion principle is presented. It is assumed that two different types of diseases transmission, say horizontal and vertical transmission, are used too. The horizontal transmission occurs by direct contact between infected and susceptible individuals, while vertical transmission occurs when the parasite is transmitted from parent to offspring [11-13]. The incidence of an epidemiological model is defined as the rate at which susceptible becomes infectious. Different types of incidence rates are introduced into literatures [14-17]. Finally two types of incidence rates, say bilinear mass action and nonlinear type, are used with the horizontal and vertical transmission, respectively. The local and global stability for all possible equilibria are carried out with the help of Lyapunov function and LaSalle's invariant principle [18]. An application of Sotomayor theorem [19, 20] for local bifurcations is used to study the occurrence of local bifurcations near the equilibria. The Hopf bifurcation [21, 22] conditions are derived. Finally, numerical simulations are used to confirm our obtained analytical results and specify the control set of parameters.

\section{Model Formulation}

Consider a real world system consisting of a host population $N(t)$ that is divided into four compartments: $S(t)$ which 
represents the number of susceptible individuals at time $t ; I_{1}(t)$ and $I_{2}(t)$ that represent the number of infected individuals at time $t$ for SIRS-type of disease and SIS-type of disease, respectively; finally $R(t)$ that represents the number of recovered individuals at time $t$, thus $N(t)=S(t)+I_{1}(t)+$ $I_{2}(t)+R(t)$. Now in order to formulate the dynamics of the above system mathematically, the following assumptions have been adopted:

(1) There is a constant number of the host populations entering to the system with recruitment rate $\Lambda>0$.

(2) There is a vertical transmission of both of the diseases; that is, the infectious host gives birth to a new infected host of rates $0 \leq p_{1} \leq 1$ and $0 \leq p_{2} \leq 1$ for the diseases $I_{1}$ and $I_{2}$, respectively. Consequently $p_{1} I_{1}$ and $p_{2} I_{2}$ individuals enter into infected compartments $I_{1}$ and $I_{2}$, respectively, and the same quantities are disappearing from recruitment in the susceptible compartment.

(3) The diseases are transmitted by contact, according to the mass action law, between the individuals in the $S$ compartment and those in $I_{i}(i=1,2)$ compartments with nonlinear incidence rate for $I_{1}$ that is given by $\beta_{1} S I_{1} /\left(1+I_{1}\right)$, in which $\beta_{1}>0$ represents the infection force rate while $1 /\left(1+I_{1}\right)$ represents the inhibition effect of the crowding effect of the infected individuals, and linear incidence rate for $I_{2}$ that is given by $\beta_{2} S I_{2}$, where $\beta_{2}>0$ represents the infection rate.

(4) The individuals in the $I_{1}$ compartment are facing death due to the disease with infection death rate $\alpha_{1} \geq$ 0 . They recover from disease and get immunity with a recovery rate $\delta>0$.

(5) The individuals in the $I_{2}$ compartment are facing death due to the disease with infection death rate $\alpha_{2} \geq$ 0 . They also recover from the disease but return back to be susceptible with recovery rate $\gamma>0$.

(6) The individuals in the $R$ compartment are losing the immunity from the $I_{1}$ disease and return back to be susceptible again with losing immunity rate $0 \leq \eta<$ 1.

(7) There is a natural death rate $\mu>0$ for the individuals in the host population. Finally, it is assumed that both the diseases cannot be transmitted to the same individual simultaneously.

According to these assumptions the dynamics of the above real world system can be represented mathematically by the following set of differential equations:

$$
\begin{aligned}
\frac{d S}{d t}= & \Lambda-\left(\frac{\beta_{1} I_{1}}{1+I_{1}}+\beta_{2} I_{2}\right) S+\left(\gamma-p_{2}\right) I_{2}-\mu S-p_{1} I_{1} \\
& +\eta R, \\
\frac{d I_{1}}{d t}= & \frac{\beta_{1} S I_{1}}{1+I_{1}}-\left(\mu+\alpha_{1}+\delta-p_{1}\right) I_{1},
\end{aligned}
$$

$$
\begin{aligned}
& \frac{d I_{2}}{d t}=\beta_{2} S I_{2}-\left(\mu+\alpha_{2}+\gamma-p_{2}\right) I_{2} \\
& \frac{d R}{d t}=\delta I_{1}-(\eta+\mu) R
\end{aligned}
$$

with the initial condition $S(0)>0, I_{1}(0)>0, I_{2}(0)>0$, and $R(0)>0$. Moreover to insure that the recruitment $\Lambda$ in the susceptible compartment is always positive the following hypotheses are assumed to be holding always:

$$
\begin{aligned}
& \delta \geq p_{1}, \\
& \gamma \geq p_{2} .
\end{aligned}
$$

Theorem 1. The closed set $\Omega=\left\{\left(S, I_{1}, I_{2}, R\right) \in \mathbb{R}_{+}^{4}: N \leq \Lambda / \mu\right\}$ is positively invariant and attracting with respect to model (1).

Proof. Let $\left(S(t), I_{1}(t), I_{2}(t), R(t)\right)$ be any solution of system (1) with any given initial condition. Then by adding all the equations in system (1) we obtain that

$$
\begin{aligned}
\frac{d N}{d t} & =\Lambda-\mu S-\left(\mu+\alpha_{1}\right) I_{1}-\left(\mu+\alpha_{2}\right) I_{2}-\mu R \\
& \leq \Lambda-\mu N .
\end{aligned}
$$

Thus, from standard comparison theorem [20], we obtain

$$
N(t) \leq N(0) e^{-\mu t}+\frac{\Lambda}{\mu}\left(1-e^{-\mu t}\right) .
$$

Consequently it is easy to verify that

$$
N(t) \leq \frac{\Lambda}{\mu}, \quad \text { when }(0) \leq \frac{\Lambda}{\mu} .
$$

Thus, $\Omega$ is positively invariant. Further, when $N(0)>\Lambda / \mu$, then either the solution enters $\Omega$ in finite time, or $N(t)$ approaches $\Lambda / \mu$ as $t \rightarrow \infty$. Hence, $\Omega$ is attracting (i.e., all solutions in $\mathbb{R}_{+}^{4}$ eventually approach, enter, or stay in $\Omega$ ).

Therefore, the system of equations given in model (1) is mathematically well-posed and epidemiologically reasonable, since all the variables remain nonnegative $\forall t \geq 0$. Further since the equations of model (1) are continuous and have continuously partial derivatives then they are Lipschitzian. In addition to that from Theorem 1, model (1) is uniformly bounded. Therefore the solution of it exists and is unique. Hence, from now onward it is sufficient to consider the dynamics of model (1) in $\Omega$.

\section{Equilibrium Points and Basic Reproduction Number}

Model (1) has four equilibrium points that are obtained by setting the right hand sides of this model equal to zero. The first equilibrium point is the disease-free equilibrium (DFE) point that is denoted by $E_{0}=\left(S_{0}, 0,0,0\right)$ with $S_{0}=\Lambda / \mu$. Moreover the basic reproduction number of model (1), which is denoted by $R_{0}$, is the maximum eigenvalue of the next 
generation matrix (i.e., the maximum of the reproduction numbers, those computed of each disease). That is,

$$
R_{0}=\max \left\{R_{1}, R_{2}\right\} .
$$

Here $R_{1}=\left(\beta_{1} S_{0}+p_{1}\right) /\left(\mu+\alpha_{1}+\delta\right)$ and $R_{2}=\left(\beta_{2} S_{0}+p_{2}\right) /(\mu+$ $\left.\alpha_{2}+\gamma\right)$.

The other three equilibrium points can be described as follows.

The first disease-free equilibrium point, which is located in the boundary $S I_{2}$-plane, is denoted by $E_{1}=\left(\bar{S}, 0, \bar{I}_{2}, 0\right)$ where

$$
\begin{aligned}
\bar{S} & =\frac{S_{0}}{T_{2}}, \\
\bar{I}_{2} & =\frac{\Lambda}{\left(\mu+\alpha_{2}\right)}\left(1-\frac{1}{T_{2}}\right),
\end{aligned}
$$

and here $T_{2}=\beta_{2} S_{0} /\left(\mu+\alpha_{2}+\gamma-p_{2}\right)$. Clearly $E_{1}$ exists uniquely in the interior of $S_{2}$-plane provided that

$$
T_{2}>1 \text {. }
$$

The second disease-free equilibrium point that is located in the boundary $S I_{1} R$-space is given by $E_{2}=\left(\widetilde{S}, \widetilde{I}_{1}, 0, \widetilde{R}\right)$ where

$$
\begin{aligned}
\widetilde{S} & =\frac{S_{0}\left(1+\widetilde{I}_{1}\right)}{T_{1}} ; \\
\widetilde{I}_{1} & =\frac{\Lambda\left(1-1 / T_{1}\right)}{\left(\mu+\alpha_{1}+\delta\right)-\eta \delta /(\eta+\mu)+\Lambda / T_{1}}, \\
\widetilde{R} & =\frac{\widetilde{I}_{1}}{\eta+\mu},
\end{aligned}
$$

and here $T_{1}=\beta_{1} S_{0} /\left(\mu+\alpha_{1}+\delta-p_{1}\right)$. Obviously $E_{2}$ exists uniquely in the interior of positive octant of $S I_{1} R$-space provided that

$$
T_{1}>1 .
$$

Finally, the endemic equilibrium point, which is denoted by $E_{3}=\left(S^{*}, I_{1}^{*}, I_{2}^{*}, R^{*}\right)$, where

$$
\begin{aligned}
& S^{*}=\frac{S_{0}}{T_{2}} \\
& I_{1}^{*}=\left(\frac{T_{1}}{T_{2}}-1\right) ;
\end{aligned}
$$

$$
\begin{aligned}
R^{*}= & \frac{\delta\left(\left(T_{1} / T_{2}\right)-1\right)}{\eta+\mu}, \\
I_{2}^{*}= & \frac{\Lambda}{\left(\mu+\alpha_{2}\right)}\left(1-\frac{1}{T_{2}}\right) \\
& -\frac{\left(\left(T_{1} / T_{2}\right)-1\right)}{\left(\mu+\alpha_{2}\right)}\left[\left(\mu+\alpha_{1}+\delta\right)-\frac{\eta \delta}{\eta+\mu}\right],
\end{aligned}
$$

exists uniquely in the interior of $\Omega$ provided that the following conditions hold:

$$
\begin{aligned}
& T_{1}>T_{2}>1 \\
& \Lambda>\frac{\Lambda}{T_{2}}+I_{1}^{*}\left(\left(\mu+\alpha_{1}+\delta\right)-\frac{\eta \delta}{\eta+\mu}\right) .
\end{aligned}
$$

Keeping the above in view, it is easy to verify with the help of condition (2) that

$$
T_{i}>1\left(T_{i}<1\right) \Longleftrightarrow R_{i}>1\left(R_{i}<1\right), \quad i=1,2 .
$$

Then directly we obtain $T_{i}>1\left(T_{i}<1\right) \Leftrightarrow R_{0}>1\left(R_{0}<\right.$ 1 ). Consequently, $T_{i}$ represent the threshold parameters for the existence of the last three equilibrium points of model (1). Moreover, it is well known that the basic reproduction number $\left(R_{0}\right)$ is representing the average number of secondary infections that occur from one infected individual in contact with susceptible individuals. Therefore if $R_{0}<1$, then each infected individual in the entire period of infectivity will produce less than one infected individual on average, which shows the disease will be wiped out of the population. However, if $R_{0}>1$, then each infected individual in the entire infection period having contact with susceptible individuals will produce more than one infected individual; this leads to the disease invading the susceptible population.

\section{Local Stability Analysis}

In this section, the local stability analyses of all possible equilibrium points of model (1) are discussed by determining the Jacobian matrix with their eigenvalues. Now the general Jacobian matrix of model (1) can be written:

$$
J=\left[\begin{array}{cccc}
-\frac{\beta_{1} I_{1}}{1+I_{1}}-\beta_{2} I_{2}-\mu & -\frac{\beta_{1} S}{\left(1+I_{1}\right)^{2}}-p_{1} & -\beta_{2} S+\left(\gamma-p_{2}\right) & \eta \\
\frac{\beta_{1} I_{1}}{1+I_{1}} & -\frac{\beta_{1} S I_{1}}{\left(1+I_{1}\right)^{2}}+f\left(S, I_{1}\right) & 0 & 0 \\
\beta_{2} I_{2} & 0 & \beta_{2} S-\left(\mu+\alpha_{2}+\gamma-p_{2}\right) & 0 \\
0 & \delta & 0 & -(\eta+\mu)
\end{array}\right] \text {, }
$$


where $f\left(S, I_{1}\right)=\beta_{1} S /\left(1+I_{1}\right)-\left(\mu+\alpha_{1}+\delta-p_{1}\right)$. Therefore the local stability results near the above equilibrium points can be presented in the following theorems.

Theorem 2. The disease-free equilibrium $E_{0}=\left(S_{0}, 0,0,0\right)$ is locally asymptotically stable when $R_{0}<1$ and unstable for $R_{0}>$ 1.

Proof. The characteristic equation of the Jacobian matrix of model (1) at the disease-free equilibrium can be written as

$$
\begin{aligned}
& (\lambda+\mu)\left(\lambda-\beta_{1} S_{0}+\left(\mu+\alpha_{1}+\delta-p_{1}\right)\right) \\
& \quad \cdot\left(\lambda-\beta_{2} S_{0}+\left(\mu+\alpha_{2}+\gamma-p_{2}\right)\right)(\lambda+(\eta+\mu))=0 .
\end{aligned}
$$

So, if $R_{0}<1$, then according to (6), (15) has four negative real roots (eigenvalues). Hence, the DFE is locally asymptotically stable. Further, for $R_{0}>1$ (15) has at least one positive eigenvalue and then the DFE is a saddle point.

Theorem 3. The first disease-free equilibrium point $E_{1}=$ $\left(\bar{S}, 0, \bar{I}_{2}, 0\right)$ of model (1) is locally asymptotically stable provided that

$$
T_{2}>1>T_{1} .
$$

Proof. The characteristic equation of the Jacobian matrix of model (1) at $E_{1}$ can be written as

$$
\begin{gathered}
{\left[\bar{\lambda}-\beta_{1} \bar{S}+\left(\mu+\alpha_{1}+\delta-p_{1}\right)\right][\bar{\lambda}+(\eta+\mu)]} \\
\cdot\left[\bar{\lambda}^{2}-\operatorname{Tr}_{1} \bar{\lambda}+D_{1}\right]=0 ;
\end{gathered}
$$

here $\operatorname{Tr}_{1}=-\left(\beta_{2} \bar{I}_{2}+\mu\right)<0$ and $D_{1}=\beta_{2} \bar{I}_{2}\left(\beta_{2} \bar{S}-(\gamma-\right.$ $\left.\left.p_{1}\right)\right)>0$ due to condition (16). Hence both the eigenvalues $\bar{\lambda}_{S}$ and $\bar{\lambda}_{I_{2}}$, which describe the dynamics in the $S$-direction and $I_{2}$-direction, respectively, have negative real parts. Moreover, from (17), the eigenvalue in the $I_{1}$-direction can be written as

$$
\bar{\lambda}_{I_{1}}=\beta_{1} \bar{S}-\left(\mu+\alpha_{1}+\delta-p_{1}\right)=\left(1-\frac{T_{2}}{T_{1}}\right) \beta_{1} \bar{S} .
$$

Thus under the given condition (16), we have $\bar{\lambda}_{I_{1}}<0$, while $\bar{\lambda}_{R}=-(\eta+\mu)$ is always negative. Hence, $E_{1}$ is locally asymptotically stable.

Theorem 4. The second disease-free equilibrium point $E_{2}=$ $\left(\widetilde{S}, \widetilde{I}_{1}, 0, \widetilde{R}\right)$ of model (1) is locally asymptotically stable provided that

$$
\begin{aligned}
& T_{1}>1>T_{2}\left(1+\widetilde{I}_{1}\right), \\
& \widetilde{P}_{2}>\delta,
\end{aligned}
$$

where $\widetilde{P}_{2}$ is given in the proof.

Proof. The characteristic equation of the Jacobian matrix of model (1) at $E_{2}$ can be written as

$$
\begin{aligned}
& {\left[\widetilde{\lambda}-\beta_{2} \widetilde{S}+\left(\mu+\alpha_{2}+\gamma-p_{2}\right)\right]\left[\tilde{\lambda}^{3}+A \tilde{\lambda}^{2}+B \widetilde{\lambda}+C\right]} \\
& \quad=0
\end{aligned}
$$

here

$$
\begin{aligned}
& A=\left(\widetilde{P}_{1}+\widetilde{P}_{2}\right)+(\eta+\mu), \\
& B=\left(\widetilde{P}_{1}+\widetilde{P}_{2}\right)(\eta+\mu)+\left(\widetilde{P}_{1} \widetilde{P}_{2}+\widetilde{P}_{3}\right), \\
& C=(\eta+\mu)\left(\widetilde{P}_{1} \widetilde{P}_{2}+\widetilde{P}_{3}\right)-\widetilde{P}_{4}
\end{aligned}
$$

with

$$
\begin{aligned}
& \widetilde{P}_{1}=\left(\frac{\beta_{1} \widetilde{I}_{1}}{1+\widetilde{I}_{1}}+\mu\right)>0, \\
& \widetilde{P}_{2}=\frac{\beta_{1} \widetilde{S}_{1}}{\left(1+\widetilde{I}_{1}\right)^{2}}>0, \\
& \widetilde{P}_{3}=\left(\frac{\beta_{1} \widetilde{I}_{1}}{1+\widetilde{I}_{1}}\left(\frac{\beta_{1} \widetilde{I}_{1}}{\left(1+\widetilde{I}_{1}\right)^{2}}+p_{1}\right)\right)>0, \\
& \widetilde{P}_{4}=\frac{\eta \delta \beta_{1} \widetilde{I}_{1}}{1+\widetilde{I}_{1}}>0 .
\end{aligned}
$$

Clearly, the eigenvalue $\widetilde{\lambda}_{I_{2}}$ in the $I_{2}$-direction can be written as

$$
\begin{aligned}
\tilde{\lambda}_{I_{2}} & =\beta_{2} \widetilde{S}-\left(\mu+\alpha_{2}+\gamma-p_{2}\right) \\
& =\left(1-\frac{T_{1}}{T_{2}\left(1+\widetilde{I}_{1}\right)}\right) \beta_{2} \widetilde{S},
\end{aligned}
$$

and thus $\widetilde{\lambda}_{I_{2}}<0$ under the condition (19a). In addition from (20) we have $A>0$ always, while $C$ can be written as

$$
\begin{aligned}
C= & \widetilde{p}_{5} \frac{\widetilde{p}_{2}}{\delta}+\mu \widetilde{p}_{5} \frac{\widetilde{p}_{2}}{\delta}+\widetilde{p}_{2}\left(\beta_{2} I_{2}^{*}(\eta+\mu)\right) \\
& +\widetilde{p}_{2} \mu(\eta+\mu)+\widetilde{p}_{3}(\eta+\mu)+\widetilde{p}_{4}\left(\widetilde{p}_{2}+(\eta+\mu)\right) \\
& -\widetilde{p}_{5} .
\end{aligned}
$$

Hence, $C>0$ provided that the sufficient condition (19b) holds. Further it is easy to verify that

$$
A B-C=\left(\widetilde{P}_{1}+\widetilde{P}_{2}\right)\left(B+(\eta+\mu)^{2}\right)+\widetilde{P}_{4}>0 .
$$

Hence, due to the Routh-Hurwitz criterion the third-degree polynomial term in (20) has roots (eigenvalues) with negative real parts. Hence $E_{2}$ is locally asymptotically stable.

Theorem 5. The endemic equilibrium point $E_{3}=$ $\left(S^{*}, I_{1}^{*}, I_{2}^{*}, R^{*}\right)$ of model (1) is locally asymptotically stable provided that

$$
\begin{aligned}
& T_{1}>T_{2}>1, \\
& \overline{\bar{Q}}>q_{5}>\delta,
\end{aligned}
$$

where $\overline{\bar{Q}}$ and $q_{5}$ are given in the proof. 
Proof. The characteristic equation of the Jacobian matrix of model (1) at $E_{3}$ can be written as

$$
\lambda^{* 4}+A_{1} \lambda^{* 3}+B_{1} \lambda^{* 2}+C_{1} \lambda^{*}+D_{1}=0 .
$$

Here

$$
\begin{aligned}
& A_{1}=\left(q_{1}+q_{2}\right)+(\eta+\mu)>0 \\
& B_{1}=(\eta+\mu)\left(q_{1}+q_{2}\right)+q_{1} q_{2}+q_{3}+q_{4}>0 \\
& C_{1}=\left(q_{1} q_{2}+q_{3}\right)(\eta+\mu)+q_{4}\left(q_{2}+(\eta+\mu)\right)-q_{5}, \\
& D_{1}=q_{4} q_{2}(\eta+\mu)>0
\end{aligned}
$$

with

$$
\begin{aligned}
& q_{1}=\frac{\beta_{1} I_{1}^{*}}{1+I_{1}^{*}}+\beta_{2} I_{2}^{*}+\mu ; \\
& q_{2}=\frac{\beta_{1} S^{*} I_{1}^{*}}{\left(1+I_{1}^{*}\right)^{2}} ; \\
& q_{3}=\frac{\beta_{1} I_{1}^{*}}{1+I_{1}^{*}}\left(\frac{\beta_{1} S^{*}}{\left(1+I_{1}^{*}\right)^{2}}+p_{1}\right), \\
& q_{4}=\beta_{2} I_{2}^{*}\left[\beta_{2} S^{*}-\left(\gamma-p_{2}\right)\right] ; \\
& q_{5}=\frac{\eta \delta \beta_{1} I_{1}^{*}}{1+I_{1}^{*}} .
\end{aligned}
$$

Obviously, $q_{i}>0, i=1,2,3,5$, while $q_{4}$ is positive under condition (26a). Now, by using the values of $q_{i}$ and the sufficient condition (26b), then straightforward computation gives

$$
C_{1}=\overline{\bar{Q}}+q_{4}\left(q_{2}+(\eta+\mu)\right)-q_{5}>0,
$$

and here $\overline{\bar{Q}}=\left(q_{1} q_{2}+q_{3}\right)(\eta+\mu)$. Moreover we have

$$
\begin{aligned}
& A_{1} B_{1} C_{1}=\left[Q+\left(q_{1}+q_{2}\right)^{2}(\eta+\mu)+q_{4} q_{1}\right. \\
& \left.+\left(q_{1} q_{2}+q_{3}+(\eta+\mu)^{2}\right)\left(q_{1}+q_{2}\right)\right]\left[Q-q_{5}\right], \\
& C_{1}^{2}+A_{1}^{2} D_{1}=\left(Q-q_{5}\right)^{2}+\left[\left(q_{1}+q_{2}\right)+(\eta+\mu)\right]^{2} \\
& \quad \cdot\left(q_{4} q_{2}(\eta+\mu)\right),
\end{aligned}
$$

where $Q=\overline{\bar{Q}}+q_{4}\left(q_{2}+(\eta+\mu)\right)$. Therefore we obtain that

$$
\begin{aligned}
A_{1} B_{1} & C_{1}-C_{1}^{2}-A_{1}^{2} D_{1} \\
= & F \overline{\bar{Q}}+q_{4} \overline{\bar{Q}}\left(q_{1}+q_{2}\right)+q_{4} q_{1} A_{1}(\eta+\mu)^{2} \\
& +q_{4}^{2} q_{1}\left(q_{2}+(\eta+\mu)\right) \\
& +q_{4} q_{2}\left(q_{1}+q_{2}\right)\left(q_{1} q_{2}+q_{3}\right)-F q_{5}+C_{1} q_{5} .
\end{aligned}
$$

Here $F=\left(q_{1}+q_{2}\right)^{2}(\eta+\mu)+\left(q_{1} q_{2}+q_{3}+(\eta+\mu)^{2}\right)\left(q_{1}+q_{2}\right)+q_{4} q_{1}$.

Hence, according to condition (26b) it is easy to verify that $A_{1} B_{1} C_{1}-C_{1}^{2}-A_{1}^{2} D_{1}>0$. Therefore, all the coefficients of (27) are positive and $A_{1} B_{1} C_{1}-C_{1}^{2}-A_{1}^{2} D_{1}>0$. Hence, due to the Routh-Hurwitz criterion all the eigenvalues $\left(\lambda_{S}^{*}, \lambda_{I_{1}}^{*}, \lambda_{I_{2}}^{*}\right.$ and $\left.\lambda_{R}^{*}\right)$ of the Jacobian matrix near the endemic equilibrium point $E_{3}$ have negative real parts. Thus, the proof is complete.

\section{Global Stability Analysis}

This section deals with the global stability of the equilibrium points of model (1) using Lyapunov methods with LaSalle's invariant principle. The obtained results are presented in the following theorems.

Theorem 6. Assume that DFE $E_{0}=\left(S_{0}, 0,0,0\right)$ of model (1) is locally asymptotically stable; then it is global asymptotically stable in $\Omega$.

Proof. Consider $\widehat{V}: \Omega \rightarrow \mathbb{R}$ that is defined by

$$
\widehat{V}\left(S, I_{1}, I_{2}, R\right)=\sum_{i=1}^{2} I_{i} .
$$

Computing the derivative of this positive semidefinite function with respect to time along the solution of model (1) and then simplifying the resulting terms give

$$
\begin{aligned}
\frac{d \widehat{V}}{d t}= & \left(\frac{\beta_{1} S}{1+I_{1}}-\left(\mu+\alpha_{1}+\delta-p_{1}\right)\right) I_{1} \\
& +\left(\beta_{2} S-\left(\mu+\alpha_{2}+\gamma-p_{2}\right)\right) I_{2} .
\end{aligned}
$$

Since the solution of model (1) is bounded by $S_{0}=\Lambda / \mu$ as $t \rightarrow \infty$,

$$
\begin{aligned}
\frac{d \widehat{V}}{d t} \leq & \left(\beta_{1} S_{0}-\left(\mu+\alpha_{1}+\delta-p_{1}\right)\right) I_{1} \\
& +\left(\beta_{2} S_{0}-\left(\mu+\alpha_{2}+\gamma-p_{2}\right)\right) I_{2} \\
= & \left(\mu+\alpha_{1}+\delta-p_{1}\right)\left(T_{1}-1\right) I_{1} \\
& +\left(\mu+\alpha_{2}+\gamma-p_{2}\right)\left(T_{2}-1\right) I_{2} .
\end{aligned}
$$

Since $T_{i}<1, i=1,2$, due to the local stability condition of $E_{0}$ then $d \widehat{V} / d t<0$. Also we have that $d \widehat{V} / d t=0$ on the set $\left\{\left(S, I_{1}, I_{2}, R\right) \in \Omega: I_{1}=I_{2}=0\right\}$, so $d \widehat{V} / d t$ is negative semidefinite and hence according to Lyapunov first theorem $E_{0}$ is globally stable point. Now, since on this set we have

$$
\frac{d S}{d t}=\Lambda-\mu S+\eta R=0,
$$

if and only if $S=S_{0}, R=0$, thus the largest invariant set contained in this set is reduced to the disease-free equilibrium point $E_{0}$. Hence according to LaSalle's invariant principle [18], $E_{0}$ is attractive point and hence it is globally asymptotically stable in $\Omega$.

Theorem 7. Assume that the first disease-free equilibrium point $E_{1}=\left(\bar{S}, 0, \bar{I}_{2}, 0\right)$ is locally asymptotically stable; then it is global asymptotically stable in $\Omega$ provided that

$$
I_{2}>\bar{I}_{2} \text {. }
$$


Proof. Consider that $L: \Omega \rightarrow \mathbb{R}$ that is defined by

$$
\begin{aligned}
L\left(S, I_{1}, I_{2}, R\right)= & \frac{1}{2}\left[(S-\bar{S})+I_{1}+\left(I_{2}-\bar{I}_{2}\right)+R\right]^{2} \\
& +\frac{\left(2 \mu+\alpha_{1}\right)}{\beta_{1}} I_{1} \\
& +\frac{\left(2 \mu+\alpha_{2}\right)}{\beta_{2}}\left(I_{2}-\bar{I}_{2}-\bar{I}_{2} \ln \frac{I_{2}}{\bar{I}_{2}}\right) \\
& +\frac{\left(2 \mu+\alpha_{1}\right)}{2 \delta} R^{2} .
\end{aligned}
$$

Clearly $L$ is continuous and positive definite function. Now by taking the derivative of $L$ with respect to time along the solution of model (1), we get after simplifying the resulting terms that

$$
\begin{aligned}
\frac{d L}{d t}= & -\mu(S-\bar{S})^{2}-\mu R^{2}-2 \mu R(S-\bar{S}) \\
& -\left(2 \mu+\alpha_{1}\right)(S-\bar{S}) I_{1} \\
& -\left(2 \mu+\alpha_{2}\right)(S-\bar{S})\left(I_{2}-\bar{I}_{2}\right) \\
& -\left(2 \mu+\alpha_{1}+\alpha_{2}\right)\left(I_{2}-\bar{I}_{2}\right) I_{1} \\
& -\left(2 \mu+\alpha_{1}\right) I_{1} R-\left(\mu+\alpha_{2}\right)\left(I_{2}-\bar{I}_{2}\right)^{2} \\
& -\left(2 \mu+\alpha_{2}\right)\left(I_{2}-\bar{I}_{2}\right) R-\left(\mu+\alpha_{1}\right) I_{1}^{2}, \\
\frac{d L}{d t} \leq & -\mu[(S-\bar{S})+R]^{2}-\mu\left[I_{1}+\left(I_{2}-\bar{I}_{2}\right)\right]^{2} \\
& -2 \mu\left(I_{2}-\bar{I}_{2}\right) R \\
& +\frac{2 \mu\left(\mu+\alpha_{1}+\delta-p_{1}\right)}{\beta_{1}}\left[\frac{T_{1}}{T_{2}}-1\right] I_{1} \\
& -\frac{\mu(\eta+\mu)}{\delta} R^{2} .
\end{aligned}
$$

Hence according to local stability condition (16) along with the sufficient condition (37) it obtains that $d L / d t$ is negative definite function. Thus due to Lyapunov second theorem $E_{1}$ is global asymptotically stable in $\Omega$.

Theorem 8. Assume that the second disease-free equilibrium point $E_{2}=\left(\widetilde{S}, \widetilde{I}_{1}, 0, \widetilde{R}\right)$ of model (1) is locally asymptotically stable; then it is global asymptotically stable in $\Omega$ if

$$
\begin{aligned}
& q_{12}^{2}<q_{11} q_{22}, \\
& q_{14}^{2}<q_{11} q_{44}, \\
& q_{23}^{2}<q_{22} q_{33}, \\
& q_{34}^{2}<q_{33} q_{44},
\end{aligned}
$$

where

$$
\begin{aligned}
& q_{11}=\mu ; \\
& q_{22}=\left(\mu+\alpha_{1}\right)+\frac{\widetilde{S}}{\left(1+\widetilde{I}_{1}\right)\left(1+I_{1}\right)} \\
& q_{33}=\left(\mu+\alpha_{2}\right) ; \\
& q_{44}=\frac{\left(2 \mu+\alpha_{1}\right)(\eta+\mu)}{2 \delta}+\mu ; \\
& q_{12}=\frac{1}{1+I_{1}}-\left(2 \mu+\alpha_{1}\right) \\
& q_{14}=2 \mu ; \\
& q_{23}=\left(2 \mu+\alpha_{1}+\alpha_{2}\right) ; \\
& q_{34}=\left(2 \mu+\alpha_{2}\right) .
\end{aligned}
$$

Proof. Consider the function $W: \Omega \rightarrow \mathbb{R}$ that is defined by

$$
\begin{aligned}
W\left(S, I_{1}, I_{2}, R\right) & \\
= & \frac{1}{2}\left[(S-\widetilde{S})+\left(I_{1}-\widetilde{I}_{1}\right)+I_{2}+(R-\widetilde{R})\right]^{2} \\
& +\frac{1}{\beta_{1}}\left(I_{1}-\widetilde{I}_{1}-\widetilde{I}_{1} \ln \frac{I_{1}}{\widetilde{I}_{1}}\right)+\frac{\left(2 \mu+\alpha_{2}\right)}{\beta_{2}} I_{2} \\
& +\frac{\left(2 \mu+\alpha_{1}\right)}{2 \delta}(R-\widetilde{R})^{2} .
\end{aligned}
$$

Clearly $W$ is continuous and positive definite function. Now by taking the derivative of $W$ with respect to time along the solution of model (1), we get after simplifying the resulting terms that

$$
\begin{aligned}
\frac{d W}{d t}= & -q_{11}(S-\widetilde{S})^{2}+q_{12}(S-\widetilde{S})\left(I_{1}-\widetilde{I}_{1}\right) \\
& -q_{14}(S-\widetilde{S})(R-\widetilde{R})-q_{22}\left(I_{1}-\widetilde{I}_{1}\right)^{2} \\
& -q_{23}\left(I_{1}-\widetilde{I}_{1}\right) I_{2}-q_{33} I_{2}^{2}-q_{34}(R-\widetilde{R}) I_{2} \\
& -q_{44}(R-\widetilde{R})^{2} \\
& +\frac{2 \mu\left(\mu+\alpha_{2}+\gamma-p_{2}\right)}{\beta_{2}}\left[\frac{T_{2}\left(1+\widetilde{I}_{1}\right)}{T_{1}}-1\right] I_{2} .
\end{aligned}
$$

Now by using the given conditions (40a)-(40d) we get that

$$
\begin{aligned}
\frac{d W}{d t} \leq & -\left[\sqrt{\frac{q_{11}}{2}}(S-\widetilde{S})-\sqrt{\frac{q_{22}}{2}}\left(I_{1}-\widetilde{I}_{1}\right)\right]^{2} \\
- & {\left[\sqrt{\frac{q_{22}}{2}}\left(I_{1}-\widetilde{I}_{1}\right)+\sqrt{\frac{q_{33}}{2}} I_{2}\right]^{2} }
\end{aligned}
$$




$$
\begin{aligned}
& -\left[\sqrt{\frac{q_{11}}{2}}(S-\widetilde{S})+\sqrt{\frac{q_{44}}{2}}(R-\widetilde{R})\right]^{2} \\
& -\left[\sqrt{\frac{q_{44}}{2}}(R-\widetilde{R})+\sqrt{\frac{q_{33}}{2}} I_{2}\right]^{2} \\
& +\frac{2 \mu\left(\mu+\alpha_{2}+\gamma-p_{2}\right)}{\beta_{2}}\left[\frac{T_{2}\left(1+\widetilde{I}_{1}\right)}{T_{1}}-1\right] I_{2} .
\end{aligned}
$$

Hence according to local stability condition (19a) it obtains that $d W / d t$ is negative definite function. Thus due to Lyapunov second theorem $E_{1}$ is global asymptotically stable in $\Omega$.

Theorem 9. Assume that the endemic equilibrium point $E_{3}=$ $\left(S^{*}, I_{1}^{*}, I_{2}^{*}, R^{*}\right)$ of model (1) is locally asymptotically stable; then it is global asymptotically stable in $\Omega$ if

$$
\begin{aligned}
& \alpha_{14}^{2}<\alpha_{11} \alpha_{44}, \\
& \alpha_{12}^{2}<\alpha_{11} \alpha_{22}, \\
& \alpha_{24}^{2}<\alpha_{22} \alpha_{44},
\end{aligned}
$$

where

$$
\begin{aligned}
& \alpha_{11}=\left(\frac{\beta_{1} I_{1}}{1+I_{1}}+\beta_{2} I_{2}+\mu\right), \\
& \alpha_{22}=\beta_{1} S^{*}, \\
& \alpha_{44}=(\eta+\mu), \\
& \alpha_{12}=\frac{\beta_{1}}{1+I_{1}}-\left(\frac{\beta_{1} S^{*}}{\left(1+I_{1}\right)\left(1+I_{1}^{*}\right)}+p_{1}\right), \\
& \alpha_{24}=\delta, \\
& \alpha_{14}=\eta .
\end{aligned}
$$

Proof. Consider the function $V: \Omega \rightarrow \mathbb{R}$ that is defined by

$$
\begin{aligned}
V\left(S, I_{1}, I_{2}, R\right)= & \frac{\left(S-S^{*}\right)^{2}}{2}+\left(I_{1}-I_{1}^{*}-I_{1}^{*} \ln \frac{I_{1}}{I_{1}^{*}}\right) \\
& +\frac{\left(2 \mu+\alpha_{2}\right)}{\beta_{2}}\left(I_{2}-I_{2}^{*}-I_{2}^{*} \ln \frac{I_{2}}{I_{2}^{*}}\right) \\
& +\frac{\left(R-R^{*}\right)^{2}}{2} .
\end{aligned}
$$

Clearly the function $V$ is continuous and positive definite function. By taking the derivative of $V$ with respect to time along the solution of model (1), we get after simplifying the resulting terms that

$$
\begin{aligned}
\frac{d V}{d t}= & -\alpha_{11}\left(S-S^{*}\right)^{2}-\alpha_{22}\left(I_{1}-I_{1}^{*}\right)^{2} \\
& -\alpha_{44}\left(R-R^{*}\right)^{2}+\alpha_{12}\left(S-S^{*}\right)\left(I_{1}-I_{1}^{*}\right) \\
& +\alpha_{14}\left(R-R^{*}\right)\left(S-S^{*}\right) \\
& +\alpha_{24}\left(I_{1}-I_{1}^{*}\right)\left(R-R^{*}\right)
\end{aligned}
$$

Then by using the given conditions (45a)-(45c) we obtain that

$$
\begin{aligned}
\frac{d V}{d t}< & -\left[\sqrt{\alpha_{11}}\left(S-S^{*}\right)+\sqrt{\alpha_{22}}\left(I_{1}-I_{1}^{*}\right)\right]^{2} \\
- & {\left[\sqrt{\alpha_{11}}\left(S-S^{*}\right)+\sqrt{\alpha_{44}}\left(R-R^{*}\right)\right]^{2} } \\
- & {\left[\sqrt{\alpha_{44}}\left(R-R^{*}\right)+\sqrt{\alpha_{22}}\left(I_{1}-I_{1}^{*}\right)\right]^{2} . }
\end{aligned}
$$

Hence, $d V / d t$ is negative semidefinite, and $d V / d t=0$ on the set $\left\{\left(S, I_{1}, I_{2}, R\right) \in \Omega: S=S^{*}, I_{1}=I_{1}^{*}, I_{2}>0, R=R^{*}\right\}$, so according to Lyapunov first theorem $E_{3}$ is globally stable point. Further, since on this set we have

$$
\begin{aligned}
\frac{d S}{d t}= & \Lambda-\left(\frac{\beta_{1} I_{1}^{*}}{1+I_{1}^{*}}+\beta_{2} I_{2}\right) S^{*}+\left(\gamma-p_{2}\right) I_{2}-\mu S^{*} \\
& -p_{1} I_{1}^{*}+\eta R^{*}=0
\end{aligned}
$$

if and only if $I_{2}=I_{2}^{*}$, then the largest compact invariant set contained in this set is reduced to the endemic equilibrium point $E_{3}$. Hence according to LaSalle's invariant principle [18], $E_{3}$ is attractive point and hence it is globally asymptotically stable in $\Omega$.

\section{Bifurcation Analysis}

In this section the local bifurcations near the equilibrium points of model (1) are investigated as shown in the following theorems with the help of Sotomayor theorem [20]. Note that model (1) can be rewritten in a vector form $d X / d t=f(X)$, where $X=\left(S, I_{1}, I_{2}, R\right)^{T}$ and $f=\left(f_{1}, f_{2}, f_{3}, f_{4}\right)^{T}$ with $f_{i}, i=1,2,3,4$, are given in the right hand side of model (1). Moreover, straightforward computation gives that the general second derivative of the Jacobian matrix (14) can be written:

$$
\begin{aligned}
& D^{2} f(X, \beta)(U, U) \\
& =\left[\begin{array}{c}
\frac{-2 \beta_{1} \zeta_{1} \zeta_{2}}{\left(1+I_{1}\right)^{2}}-2 \beta_{2} \zeta_{1} \zeta_{3}+\frac{2 \beta_{1} S\left(\zeta_{2}\right)^{2}}{\left(1+I_{1}\right)^{3}} \\
\frac{2 \beta_{1} \zeta_{1} \zeta_{2}}{\left(1+I_{1}\right)^{2}}-\frac{2 \beta_{1} S\left(\zeta_{2}\right)^{2}}{\left(1+I_{1}\right)^{3}} \\
2 \beta_{2} \zeta_{1} \zeta_{3} \\
0
\end{array}\right],
\end{aligned}
$$

where $\beta$ is any bifurcation parameter and $U=\left(\zeta_{1}, \zeta_{2}, \zeta_{3}, \zeta_{4}\right)^{T}$ is any eigenvector. 
Theorem 10. Assume that $T_{2}<1$; then as $T_{1}$ passes through the value $T_{1}=1$, model (1) near the disease-free equilibrium $E_{0}$ has

(1) no saddle-node bifurcation;

(2) a transcritical bifurcation;

(3) no pitchfork bifurcation.

Proof. Since $T_{1}=\beta_{1} S_{0} /\left(\mu+\alpha_{1}+\delta-p_{1}\right)=1$; then $\beta_{1}=$ $\left(\mu+\alpha_{1}+\delta-p_{1}\right) / S_{0}=\beta_{1}^{*}$. Now straightforward computation shows that the Jacobian matrix of model (1) at $E_{0}$ with $\beta_{1}=\beta_{1}^{*}$ has zero eigenvalue $\left(\lambda_{I_{1}}=0\right)$ and can be written as follows:

$$
\begin{aligned}
& J_{0} \\
& =\left[\begin{array}{cccc}
-\mu & -\beta_{1}^{*} S_{0}-p_{1} & -\beta_{2} S_{0}+\left(\gamma-p_{2}\right) & \eta \\
0 & 0 & 0 & 0 \\
0 & 0 & \beta_{2} S_{0}-\left(\mu+\alpha_{2}+\gamma-p_{2}\right) & 0 \\
0 & \delta & 0 & -(\eta+\mu)
\end{array}\right] .
\end{aligned}
$$

Let $V=\left(v_{1}, v_{2}, v_{3}, v_{4}\right)^{T}$ be the eigenvector corresponding to $\lambda_{I_{1}}=0$. Thus $J_{0} V=0$ gives

$$
V=\left[\begin{array}{c}
a v_{2} \\
v_{2} \\
0 \\
b v_{2}
\end{array}\right]
$$

where $v_{2}$ is any nonzero real number, $a=(\eta \delta /(\eta+\mu)-(\mu+$ $\left.\left.\alpha_{1}+\delta\right)\right) / \mu$, and $b=\delta /(\eta+\mu)$.

Similarly, $W=\left(w_{1}, w_{2}, w_{3}, w_{4}\right)^{T}$ represents the eigenvector corresponding to eigenvalue $\lambda_{I_{1}}=0$ of $J_{0}^{T}$. Hence $J_{0}^{T} W=0$ gives that

$$
W=\left[\begin{array}{c}
0 \\
w_{2} \\
0 \\
0
\end{array}\right]
$$

and here $w_{2}$ is any nonzero real number. Now, since

$$
\frac{d f}{d \beta_{1}}=f_{\beta_{1}}\left(X, \beta_{1}\right)=\left(\frac{-I_{1} S}{1+I_{1}}, \frac{I_{1} S}{1+I_{1}}, 0,0\right)^{T} \text {, }
$$

thus $f_{\beta_{1}}\left(E_{0}, \beta_{1}^{*}\right)=(0,0,0,0)^{T}$, which gives $W^{T} f_{\beta_{1}}\left(E_{0}, \beta_{1}^{*}\right)=$ 0 .

$$
J_{1}=\left[\begin{array}{cccc}
-\beta_{2} \bar{I}_{2}-\mu & -\beta_{1}^{* *} \bar{S}-p_{1} & -\beta_{2} \bar{S}+\left(\gamma-p_{2}\right) & \eta \\
0 & 0 & 0 & 0 \\
\beta_{2} \bar{I}_{2} & 0 & \beta_{2} \bar{S}-\left(\mu+\alpha_{2}+\gamma-p_{2}\right) & 0 \\
0 & \delta & 0 & -(\eta+\mu)
\end{array}\right]
$$
cation, model (1) has no saddle-node bifurcation near DFE at $\beta_{1}=\beta_{1}^{*}$.

Now since

$$
D f_{\beta_{1}}\left(E_{0}, \beta_{1}^{*}\right)=\left[\begin{array}{cccc}
0 & -S_{0} & 0 & 0 \\
0 & S_{0} & 0 & 0 \\
0 & 0 & 0 & 0 \\
0 & 0 & 0 & 0
\end{array}\right],
$$

then, $W^{T}\left(D f_{\beta_{1}}\left(E_{0}, \beta_{1}^{*}\right) V\right)=S_{0} v_{2} w_{2} \neq 0$. Now, by substituting $E_{0}$ and $\beta_{1}^{*}$ in (51) we get

$$
\begin{aligned}
& D^{2} f\left(E_{0}, \beta_{1}^{*}\right) \cdot(V, V) \\
& \quad=\left[\begin{array}{c}
-2 \beta_{1}^{*} v_{1} v_{2}-2 \beta_{2} v_{1} v_{3}+2 \beta_{1}^{*} S_{0}\left(v_{2}\right)^{2} \\
2 \beta_{1}^{*} v_{1} v_{2}-2 \beta_{1}^{*} S_{0}\left(v_{2}\right)^{2} \\
2 \beta_{2} v_{1} v_{3} \\
0
\end{array}\right] .
\end{aligned}
$$

Therefore,

$$
\begin{aligned}
W^{T} & \left(D^{2} f\left(E_{0}, \beta_{1}^{*}\right) \cdot(V, V)\right) \\
& =\left(2 \beta_{1}^{*} v_{1} v_{2}-2 \beta_{1}^{*} S_{0}\left(v_{2}\right)^{2}\right) w_{2} \\
& =\left[2 \beta_{1}^{*}\left(v_{2}\right)^{2}\left(a-S_{0}\right)\right] w_{2} \neq 0 .
\end{aligned}
$$

So, according to Sotomayor's theorem model (1) has a transcritical bifurcation at $E_{0}$ with parameter $\beta_{1}=\beta_{1}^{*} \approx$ $\left(T_{1}=1\right)$, while the pitchfork bifurcation cannot occur.

Note that similar results as those of Theorem 10 are obtained at $T_{2}=1$ or $\beta_{2}=\beta_{2}^{*}=\left(\mu+\alpha_{2}+\gamma-p_{2}\right) / S_{0}$.

Theorem 11. Assume that $T_{1}=T_{2}$; then model (1) near the first disease-free equilibrium point $E_{1}=\left(\bar{S}, 0, \bar{I}_{2}, 0\right)$ has

(1) no saddle-node bifurcation;

(2) a transcritical bifurcation;

(3) no pitchfork bifurcation.

Proof. There are two cases; in the first case, it is assumed that $T_{1}=T_{2}=1$; then straightforward computation shows that $J\left(E_{1}\right)=J\left(E_{0}\right)$; that is, $\bar{I}_{2}=0$. So (by Theorem 10) model (1) has no bifurcation and then the proof is complete.

Now in the second case it is assumed that $T_{1}=T_{2} \neq 1$ or equivalently $\beta_{1}=\beta_{1}^{* *}=\beta_{2}\left(\mu+\alpha_{1}+\delta-p_{1}\right) /\left(\mu+\alpha_{2}+\gamma-p_{2}\right)$. So straightforward computation shows that the Jacobian matrix of model (1) at $E_{1}$ with $\beta_{1}=\beta_{1}^{* *}$ has zero eigenvalue $\left(\lambda_{I_{1}}=0\right)$ and can be written as follows:
Thus, according to Sotomayor's theorem for local bifur- 
Let $X=\left(x_{1}, x_{2}, x_{3}, x_{4}\right)^{T}$ be the eigenvector corresponding to $\lambda_{I_{1}}=0$, which satisfies $J_{1} X=0$, so we get

$$
X=\left[\begin{array}{c}
0 \\
x_{2} \\
a_{1} x_{2} \\
b x_{2}
\end{array}\right],
$$

where $x_{2}$ is any nonzero real number, $a_{1}=-\left[\left(\beta_{1} \bar{S}+p_{1}\right)-\right.$ $\eta \delta /(\eta+\mu)] / \beta_{1} \bar{S}-\left(\gamma-p_{2}\right)$, and $b=\delta /(\eta+\mu)$.

Similarly the eigenvector $Y=\left(y_{1}, y_{2}, y_{3}, y_{4}\right)^{T}$ that is corresponding to the eigenvalue $\lambda_{I_{1}}=0$ of $J_{1}^{T}$ satisfies $J_{1}^{T} Y=$ 0 , so we get

$$
Y=\left[\begin{array}{c}
0 \\
y_{2} \\
0 \\
0
\end{array}\right],
$$

and here $y_{2}$ is any nonzero real number. Now, since

$$
\frac{d f}{d \beta_{1}}=f_{\beta_{1}}\left(X, \beta_{1}\right)=\left(\frac{-I_{1} S}{1+I_{1}}, \frac{I_{1} S}{1+I_{1}}, 0,0\right)^{T}
$$

then by substituting the values of $E_{1}$ and $\beta_{1}^{* *}$ we obtain that $f_{\beta_{1}}\left(E_{1}, \beta_{1}^{* *}\right)=(0,0,0,0)^{T}$ and hence we get that $Y^{T} f_{\beta_{1}}\left(E_{1}, \beta_{1}^{* *}\right)=0$.

Thus according to Sotomayor's theorem for local bifurcation, model (1) has no saddle-node bifurcation near $E_{1}$ at $\beta_{1}=\beta_{1}^{* *}$. Now since

$$
D f_{\beta_{1}}\left(E_{1}, \beta_{1}\right)=\left[\begin{array}{cccc}
0 & -\bar{S} & 0 & 0 \\
0 & \bar{S} & 0 & 0 \\
0 & 0 & 0 & 0 \\
0 & 0 & 0 & 0
\end{array}\right],
$$

then $Y^{T}\left(D f_{\beta_{1}}\left(E_{1}, \beta_{1}^{* *}\right) X\right)=\bar{S} x_{2} y_{2} \neq 0$. Thus by substituting $E_{1}$ and $\beta_{1}^{* *}$ in (51) we get

$$
\begin{aligned}
& D^{2} f\left(E_{1}, \beta_{1}^{* *}\right) \cdot(X, X) \\
& \quad=\left[\begin{array}{c}
-2 \beta_{1}^{* *} x_{1} x_{2}-2 \beta_{2} x_{1} x_{3}+2 \beta_{1}^{* *} \bar{S}\left(x_{2}\right)^{2} \\
2 \beta_{1}^{* *} x_{1} x_{2}-2 \beta_{1}^{* *} \bar{S}\left(x_{2}\right)^{2} \\
2 \beta_{2} x_{1} x_{3} \\
0
\end{array}\right] .
\end{aligned}
$$

Therefore, $\left[Y^{T}\left(D^{2} f\left(E_{1}, \beta_{1}^{* *}\right) \cdot(X, X)\right)\right]=-2 \beta_{1}^{* *} \bar{S}\left(x_{2}\right)^{2} y_{2} \neq 0$.

So, model (1) has a transcritical bifurcation at $E_{1}$ with parameter $\beta_{1}=\beta_{1}^{* *} \approx\left(T_{1}=T_{2}=1\right)$, while the pitchfork bifurcation cannot occur and hence the proof is complete.

Theorem 12. Assume that condition (19b) holds and let $T_{1}=$ $T_{2}\left(1+\widetilde{I}_{1}\right)$; then model (1) near the second disease-free equilibrium point $E_{2}=\left(\widetilde{S}, \widetilde{I}_{1}, 0, \widetilde{R}\right)$ undergoes

(1) no saddle-node bifurcation;

(2) a transcritical bifurcation;

(3) no pitchfork bifurcation.
Proof. From $T_{1}=T_{2}\left(1+\widetilde{I}_{1}\right)$ it is obtained that

$$
\beta_{2}=\beta_{2}^{* *}=\frac{\beta_{1}\left(\mu+\alpha_{2}+\gamma-p_{2}\right)}{\left(\mu+\alpha_{1}+\delta-p_{1}\right)\left(1+\widetilde{I}_{1}\right)},
$$

and then straightforward computation shows that the Jacobian matrix of model (1) at $E_{2}$ with $\beta_{2}=\beta_{2}^{* *}$ has zero eigenvalue $\left(\lambda_{I_{2}}=0\right)$ and can be written as follows:

$$
\begin{aligned}
& J_{2} \\
& =\left[\begin{array}{cccc}
\frac{-\beta_{1} \widetilde{I}_{1}}{1+\widetilde{I}_{1}}-\mu & \frac{-\beta_{1} \widetilde{S}}{\left(1+\widetilde{I}_{1}\right)^{2}}-p_{1} & -\beta_{2}^{* *} \widetilde{S}+\left(\gamma-p_{2}\right) & \eta \\
\frac{\beta_{1} \widetilde{I}_{1}}{1+\widetilde{I}_{1}} & \frac{-\beta_{1} \widetilde{S}_{1}}{\left(1+\widetilde{I}_{1}\right)^{2}} & 0 & 0 \\
0 & 0 & 0 & 0 \\
0 & \delta & 0 & -(\eta+\mu)
\end{array}\right] .
\end{aligned}
$$

Further the eigenvector $L=\left(l_{1}, l_{2}, l_{3}, l_{4}\right)^{T}$ that is corresponding to $\lambda_{I_{2}}=0$ satisfies $J_{2} L=0$, so we get

$$
L=\left[\begin{array}{c}
\frac{\widetilde{S}}{1+\widetilde{I}_{1}} l_{2} \\
l_{2} \\
\frac{-E}{\beta_{2}^{* *} \tilde{S}-\left(\gamma-p_{2}\right)} l_{2} \\
b l_{2}
\end{array}\right] \text {, }
$$

where $l_{2}$ is any nonzero real number and $E=\left(\beta_{1} \widetilde{S} /(1+\right.$ $\left.\left.\widetilde{I}_{1}\right)^{2}\right)\left(\widetilde{I}_{1}+1\right)+\mu \widetilde{S} / 1+\widetilde{I}_{1}+p_{1}-\eta b$.

Similarly the eigenvector $K=\left(k_{1}, k_{2}, k_{3}, k_{4}\right)^{T}$ that is corresponding to eigenvalue $\lambda_{I_{2}}=0$ of $J_{2}^{T}$ satisfies $J_{2}^{T} K=0$, so we get

$$
K=\left[\begin{array}{c}
0 \\
0 \\
k_{3} \\
0
\end{array}\right]
$$

and here $k_{3}$ is any nonzero real number. Now, since $d f / d \beta_{2}=$ $f_{\beta_{2}}\left(X, \beta_{2}\right)=\left(-I_{2} S, I_{2} S, 0,0\right)^{T}$, therefore $f_{\beta_{2}}\left(E_{2}, \beta_{2}^{* *}\right)=$ $(0,0,0,0)^{T}$, which yields $K^{T} f_{\beta_{2}}\left(E_{2}, \beta_{2}^{* *}\right)=0$. Consequently according to Sotomayor's theorem for local bifurcation, model (1) has no saddle-node bifurcation near $E_{2}$ at $\beta_{2}=$ $\beta_{2}^{* *}$.

Now since

$$
D f_{\beta_{2}}\left(E_{2}, \beta_{2}\right)=\left[\begin{array}{cccc}
0 & 0 & -\widetilde{S} & 0 \\
0 & 0 & 0 & 0 \\
0 & 0 & \widetilde{S} & 0 \\
0 & 0 & 0 & 0
\end{array}\right]
$$


then $K^{T}\left(D f_{\beta_{2}}\left(E_{2}, \beta_{2}^{* *}\right) L\right)=\widetilde{S} l_{3} k_{3} \neq 0$. Now, by substituting $E_{2}$ and $\beta_{2}^{* *}$ in (51) we get

$$
\begin{aligned}
& D^{2} f\left(E_{2}, \beta_{2}^{* *}\right) \cdot(L, L) \\
& =\left[\begin{array}{c}
\frac{-\beta_{1}}{\left(1+\widetilde{I}_{1}\right)^{2}} l_{1} l_{2}-2 \beta_{2}^{* *} l_{1} l_{3}-\frac{\beta_{1} l_{1} l_{2}}{\left(1+\widetilde{I}_{1}\right)^{2}}+\frac{2 \beta_{1} S\left(l_{2}\right)^{2}}{\left(1+\widetilde{I}_{1}\right)^{3}} \\
\frac{\beta_{1} l_{2}}{\left(1+\widetilde{I}_{1}\right)^{2}} l_{1} l_{2}+\frac{\beta_{1}}{\left(1+\widetilde{I}_{1}\right)^{2}} l_{1} l_{2}-\frac{2 \beta_{1} S\left(l_{2}\right)^{2}}{\left(1+\widetilde{I}_{1}\right)^{3}} \\
2 \beta_{2}^{* *} l_{1} l_{3} \\
0
\end{array}\right] .
\end{aligned}
$$

Therefore, $\left[K^{T}\left(D^{2} f\left(E_{2}, \beta_{2}^{* *}\right) \cdot(L, L)\right)\right]=2 \beta_{2}^{* *} l_{1} l_{3} k_{3} \neq 0$. Thus model (1) undergoes a transcritical bifurcation at $E_{2}$ with parameter $\beta_{2}=\beta_{2}^{* *} \approx\left(T_{1}=T_{2}\left(1+\widetilde{I}_{1}\right)\right)$, while the pitchfork bifurcation cannot occur.

Moreover, the following results are obtained too:

(1) Although $T_{1}=T_{2} \neq 1$ gives $\beta_{2}=\grave{\beta}_{2}^{* *}$, model (1) does not undergo any of the above types of bifurcation near the equilibrium point $E_{1}$ with parameter $\beta_{2}=\grave{\beta}_{2}^{* *}$.

(2) Although $T_{1}=T_{2}\left(1+\widetilde{I}_{1}\right)$ gives $\beta_{1}=\grave{\beta}_{1}^{* *}$, model (1) does not undergo any of the above types of bifurcation near the equilibrium point $E_{2}$ with parameter $\beta_{1}=$ $\grave{\beta}_{1}^{* *}$.

(3) The determinant of the Jacobian matrix at $E_{3}$, say $J\left(E_{3}\right)$, cannot be zero and hence it has no real zero eigenvalue. So there is no bifurcation near $E_{3}$.

Keeping the above in view, in the following theorem we detect of the possibility of having Hopf bifurcation.

Theorem 13. Assume that condition (26a) holds and let the following conditions be satisfied. Then model (1) undergoes Hopf bifurcation around the endemic equilibrium point when the parameter $\beta_{2}$ crosses a critical positive value $\grave{\beta}_{2}^{*}$,

$$
\begin{aligned}
N_{2} & >0, \\
N_{4} & <0, \\
\sigma_{1} & >2 \sigma_{2}, \\
\delta & <q_{5}<\left\{q_{4} q_{2}, q_{4} q_{1}\right\}, \\
q_{2} & >1,
\end{aligned}
$$

and here $\sigma_{1}=q_{1}^{2}+q_{2}^{2}+(\eta+\mu)^{2}$ and $\sigma_{2}=\left(q_{1}+q_{2}\right)(\eta+\mu)+$ $2 q_{1} q_{2}+2 q_{3}+2 q_{4}$, while $N_{2}$ and $N_{4}$ are given in the proof.

Proof. It is well known that, in order for Hopf bifurcation in four-dimensional systems to occur, the following conditions should be satisfied [21, 22]:

(1) The characteristic equation given in (28) has two real and negative eigenvalues and two complex eigenvalues, say, $\lambda^{*}\left(\beta_{2}\right)=\tau_{1}\left(\beta_{2}\right) \pm \tau_{2}\left(\beta_{2}\right)$.
(2) $\tau_{1}\left(\grave{\beta}_{2}^{*}\right)=0$.

(3) $\left.\left(d / d \beta_{2}\right) \tau_{1}\left(\beta_{2}\right)\right|_{\beta_{2}=\dot{\beta}_{2}^{*}} \neq 0$ (The transversality condition).

Accordingly the first two points are satisfied if and only if

$$
\begin{aligned}
& \Delta_{2}\left(\grave{\beta}_{2}^{*}\right)=A_{1} B_{1} C_{1}-C_{1}^{2}-A_{1}^{2} D_{1}=0, \\
& A_{1}^{3}-4 \Delta_{1}>0,
\end{aligned}
$$

while the third condition holds provided that

$$
\begin{aligned}
& \left.\frac{d}{d \beta_{2}} \tau_{1}\left(\beta_{2}\right)\right|_{\beta_{2}=\dot{\beta}_{2}^{*}} \\
& =\frac{-\left(\Psi\left(\beta_{2}\right) \Theta\left(\beta_{2}\right)+\Phi\left(\beta_{2}\right) \Gamma\left(\beta_{2}\right)\right)}{\Psi\left(\beta_{2}\right)^{2}+\Phi\left(\beta_{2}\right)^{2}} \neq 0 .
\end{aligned}
$$

That means $\Psi\left(\beta_{2}\right) \Theta\left(\beta_{2}\right)+\Phi\left(\beta_{2}\right) \Gamma\left(\beta_{2}\right) \neq 0$.

Here

$$
\begin{aligned}
\Psi\left(\beta_{2}\right)= & \left(4 \tau_{1}^{3}-12 \tau_{1} \tau_{2}^{2}\right)+3 A_{1}\left(\tau_{1}^{2}-\tau_{2}^{2}\right)+2 B_{1} \tau_{1} \\
& +C_{1} \\
\Phi\left(\beta_{2}\right)= & \left(12 \tau_{1}^{2} \tau_{2}-4 \tau_{2}^{3}\right)+6 A_{1} \tau_{1} \tau_{2}+2 B_{1} \tau_{2} \\
\Theta\left(\beta_{2}\right)= & \dot{A_{1}}\left(\tau_{1}^{3}-3 \tau_{1} \tau_{2}^{2}\right)+\dot{B_{1}}\left(\tau_{1}^{2}-\tau_{2}^{2}\right)+\dot{C}_{1} \tau_{1} \\
& +\dot{D}_{1}, \\
\Gamma\left(\beta_{2}\right)= & \dot{A_{1}}\left(3 \tau_{1}^{2} \tau_{2}-\tau_{2}^{3}\right)+2 \dot{B}_{1} \tau_{1} \tau_{2}+\dot{C}_{1} \tau_{2} .
\end{aligned}
$$

Now, straightforward computation shows the condition

$$
\Delta_{2}\left(\grave{\beta}_{2}^{*}\right)=0
$$

gives that $N_{1} \beta_{2}^{3}+N_{2} \beta_{2}^{2}+N_{3} \beta_{2}+N_{4}=0$,

where

$$
\begin{aligned}
& N_{1}=\left[q_{2}(\eta+\mu)\left((\eta+\mu)+q_{2}\right)+(\eta+\mu)\left(\mu+\alpha_{2}\right)((\eta\right. \\
& \left.\left.\quad+\mu)+2 q_{2}+\left(\mu+\alpha_{2}\right)\right)\right] I_{2}^{* 3}>0, \\
& N_{2}=\left[( \eta + \mu ) \left[q_{2}(\eta+\mu)\left(3 q_{6}+2+(\eta+\mu)\right)\right.\right. \\
& \quad+q_{3}\left((\eta+\mu)+2\left(\mu+\alpha_{2}\right)+2 q_{2}\right)+\left(\mu+\alpha_{2}\right) \\
& \quad+\left[q_{6}\left(2(\eta+\mu)+\left(\mu+\alpha_{2}\right)\right)\right. \\
& \left.\quad+q_{2}\left((\eta+\mu)+4 q_{6}+q_{2}\right)\right]+q_{2}^{2}\left(3 q_{6}+q_{2}\right)+(\mu \\
& \left.\left.\quad+\alpha_{2}\right)(\eta+\mu)^{2}-q_{5}\right]+\left(\mu+\alpha_{2}\right)\left[q_{2}^{2}\left(1+q_{2}\right)\right. \\
& \left.\left.\quad+q_{2}\left(q_{3}+q_{6}\left(\mu+\alpha_{2}\right)\right)\right]-q_{2} q_{5}\right] I_{2}^{* 2},
\end{aligned}
$$




$$
\begin{aligned}
N_{3} & =\left[( \eta + \mu ) ^ { 2 } \left[q_{2}^{3}+q_{3}\left(2 q_{6}+2 q_{2}+(\eta+\mu)\right)\right.\right. \\
& \left.+3 q_{6} q_{2}-q_{5}+\left(q_{6}+q_{2}\right) q_{6}\left(\mu+\alpha_{2}\right)\right]+(\eta+\mu) \\
& \cdot\left[q_{6} q_{2}^{2}\left(3 q_{6}+2 q_{2}\right)+\left(2 q_{6}+q_{2}\right)\left[2 q_{2} q_{3}\right.\right. \\
& \left.+q_{2}(\eta+\mu)^{2}\right]+\left(\mu+\alpha_{2}\right)\left[1+q_{6}(\eta+\mu)^{2}\right. \\
& \left.\left.+\left(2 q_{6}+q_{2}\right)\left(q_{6} q_{2}+q_{3}-2 q_{6} q_{5}\right)-q_{2} q_{5}\right]\right]+q_{2}(\mu \\
& \left.+\alpha_{2}\right)\left[q_{6} q_{2}\left(1+q_{2}\right)+\left(q_{6}+q_{2}\right) q_{3}+1\right]-\left(q _ { 2 } \left(2 q_{6}\right.\right. \\
& \left.\left.\left.+q_{2}\right)+q_{3}\right) q_{5}\right] I_{2}^{*}, \\
N_{4} & =\left[( \eta + \mu ) ^ { 2 } \left[q_{6} q_{2}\left(q_{6}^{2}+q_{6} q_{2}+q_{2}^{2}\right)+q_{3} q_{6}\left(q_{6}\right.\right.\right. \\
& \left.+2 q_{2}\right)+\left(q_{6}+q_{2}\right)(\eta+\mu)\left(q_{6} q_{2}+q_{3}\right)+q_{3} q_{2}^{2} \\
& \left.-q_{2}\right]+\left(q_{6}+q_{2}\right)(\eta+\mu)\left[q_{6}^{2} q_{2}^{2}+2 q_{6} q_{2} q_{3}+q_{3}^{2}\right] \\
& +\left((\eta+\mu)\left(q_{3}-q_{6} q_{2}\right)-q_{6}(\eta+\mu)\left(q_{6}+(\eta+\mu)\right)\right. \\
& \left.\left.-q_{2}^{2}(\eta+\mu)-\left(q_{6}+q_{2}\right)\left(q_{6} q_{2}+q_{3}\right)\right) q_{5}-q_{5}^{2}\right]
\end{aligned}
$$

with $q_{1}, q_{2}, q_{3}, q_{4}$, and $q_{5}$ given in (28) and $q_{6}=q_{1}-\beta_{2} I_{2}^{*}$. Clearly, from condition (71a) there is unique positive root, say, $\beta_{2}=\grave{\beta}_{2}{ }^{*}$. Consequently by using $\Delta_{2}=0$ in the characteristic equation and then doing some algebraic computation we get four roots,

$$
\begin{aligned}
\lambda_{1,2}\left(\grave{\beta}_{2}^{*}\right) & = \pm i \sqrt{\frac{C_{1}}{A_{1}}}=\tau_{2}\left(\grave{\beta}_{2}^{*}\right), \\
\lambda_{3,4} & =\frac{1}{2}\left(-A_{1} \pm \sqrt{A_{1}^{2}-4 \frac{\Delta_{1}}{A_{1}}}\right) .
\end{aligned}
$$

Now, it is easy to verify that $\lambda_{3}$ and $\lambda_{4}$ are real and negative provided that $(71 b)$.

Further for $\beta_{2} \in\left(\grave{\beta}_{2}^{*}-\epsilon, \grave{\beta}_{2}^{*}+\epsilon\right)$ the general form of complex eigenvalues can be written as

$$
\begin{aligned}
& \lambda_{1}=\tau_{1}\left(\beta_{2}\right)+i \tau_{2}\left(\beta_{2}\right), \\
& \lambda_{2}=\tau_{1}\left(\beta_{2}\right)-i \tau_{2}\left(\beta_{2}\right) .
\end{aligned}
$$

Substituting $\lambda\left(\beta_{2}\right)=\tau_{1}\left(\beta_{2}\right)+i \tau_{2}\left(\beta_{2}\right)$ into characteristic equation and after that calculating the derivative with respect to $\beta_{2}$ and then comparing the real and imaginary parts give that

$$
\begin{aligned}
& \Psi\left(\beta_{2}\right) \dot{\tau}_{1}\left(\beta_{2}\right)-\Phi\left(\beta_{2}\right) \dot{\tau}_{2}\left(\beta_{2}\right)=-\Theta\left(\beta_{2}\right), \\
& \Phi\left(\beta_{2}\right) \dot{\tau}_{1}\left(\beta_{2}\right)+\Psi\left(\beta_{2}\right) \dot{\tau}_{2}\left(\beta_{2}\right)=-\Gamma\left(\beta_{2}\right) .
\end{aligned}
$$

Moreover, by solving the above linear system for $\dot{\tau}_{1}\left(\beta_{2}\right)$ and $\dot{\tau}_{2}\left(\beta_{2}\right)$ then we get that

$$
\begin{aligned}
\Psi & \left(\beta_{2}\right) \Theta\left(\beta_{2}\right)+\Phi\left(\beta_{2}\right) \Gamma\left(\beta_{2}\right)=q_{1} q_{2}\left[q_{3}\left(\mu+\alpha_{2}\right)\right. \\
& +2(\eta+\mu)^{3} \\
& \left.+\left(\mu+\alpha_{2}\right)(\eta+\mu)\left((\eta+\mu)+\left(3 q_{2}+3 q_{1}-1\right)\right)\right] \\
& +q_{2} q_{3}\left[q_{2}\left(\mu+\alpha_{2}\right)\right. \\
& \left.+(\eta+\mu)\left(\left(\mu+\alpha_{2}\right)+\left(2 q_{2}+2 q_{1}-1\right)\right)\right]+q_{1}(\eta \\
& +\mu)\left(\mu+\alpha_{2}\right)\left[(\eta+\mu)+2 q_{3}+(\eta+\mu)^{2}+q_{4}\right]+(\eta \\
& +\mu)^{3}\left[q_{2}\left(\mu+\alpha_{2}\right)+q_{2}^{2}+q_{3}+q_{4}\right]+q_{5}\left(\mu+\alpha_{2}\right) \\
& \cdot\left[2 q_{2}+(\eta+\mu)\right]+q_{1} q_{3}(\eta+\mu)^{2}+q_{2}(\eta+\mu)(\mu \\
+ & \left.\alpha_{2}\right)\left[q_{2}\left(q_{2}-1\right)+(\eta+\mu)\left(2 q_{2}+2 q_{1}-1\right)\right] \\
+ & q_{1}\left(\mu+\alpha_{2}\right)\left[q_{1} q_{2}^{2}+q_{2}^{3}+q_{2} q_{4}-q_{5}\right]+q_{1}(\eta+\mu) \\
& \cdot\left[2 q_{1} q_{2}\left(1+q_{2}\right)+2 q_{2}^{3}+q_{2} q_{4}-q_{5}\right]+\left(q_{2} q_{4}-q_{5}\right) \\
& \cdot\left(q_{1} q_{2}+q_{2}^{2}\right)+(\eta+\mu)^{2}\left[3 q_{1} q_{2}^{2}+q_{2}^{3}+q_{1} q_{4}-q_{5}\right] .
\end{aligned}
$$

Thus it is easy to verify that $\Psi\left(\beta_{2}\right) \Theta\left(\beta_{2}\right)+\Phi\left(\beta_{2}\right) \Gamma\left(\beta_{2}\right) \neq 0$ provided that (71c) and (71d). Thus, the proof is complete.

\section{Numerical Simulations}

In this section, the global dynamics of model (1) is investigated numerically for different sets of initial values and different sets of parameters values. The objectives of such investigation are to determine the effect of varying the parameters values and confirm our obtained results. It is observed that, for the following biologically feasible set of hypothetical parameters values

$$
\begin{aligned}
\Lambda & =20, \\
\beta_{1} & =0.75, \\
\beta_{2} & =0.1, \\
\gamma & =0.75, \\
p_{1} & =0.01, \\
p_{2} & =0.01 \\
\mu & =0.3, \\
\eta & =0.5, \\
\alpha_{1} & =0.1, \\
\alpha_{2} & =0.6, \\
\delta & =0.7,
\end{aligned}
$$



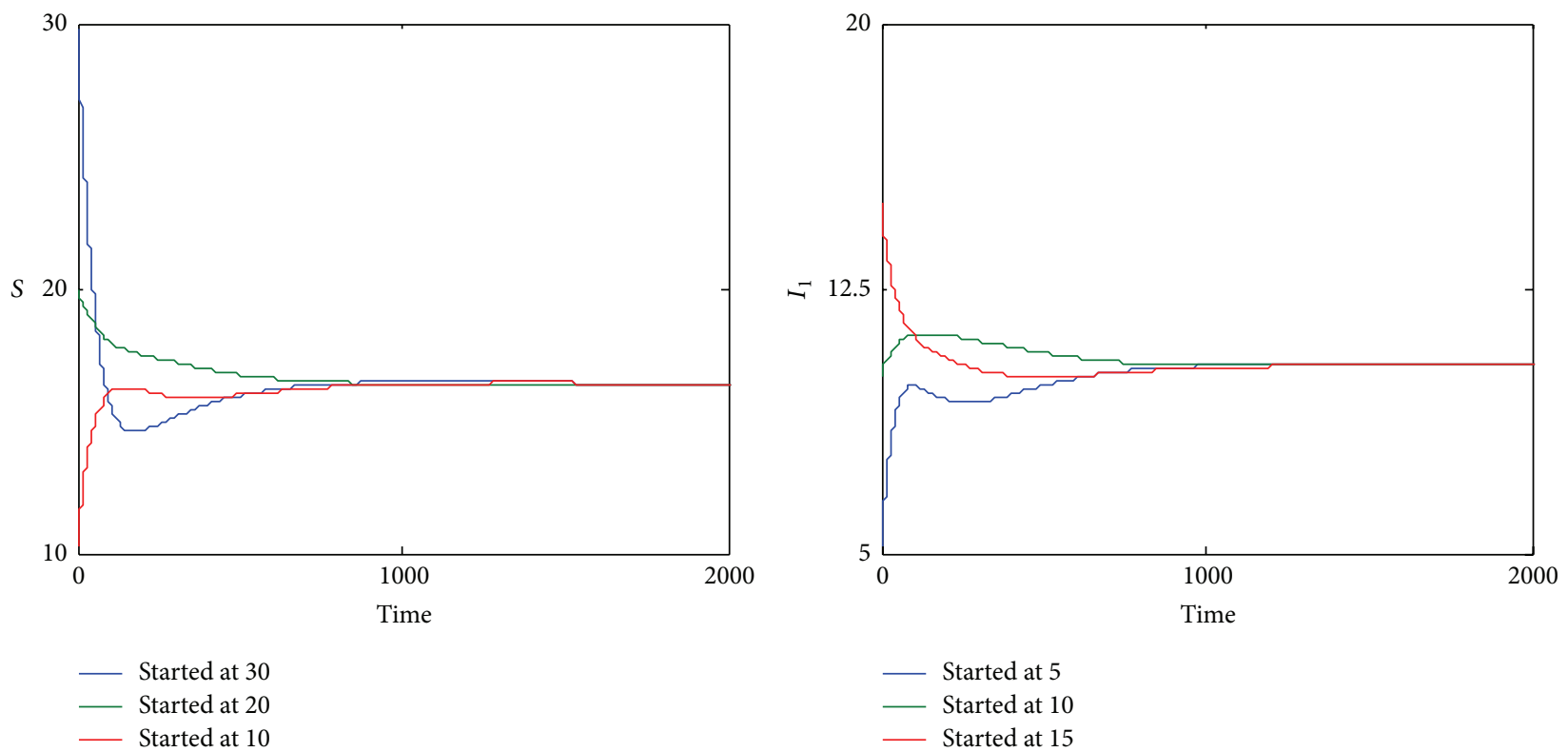

(a)

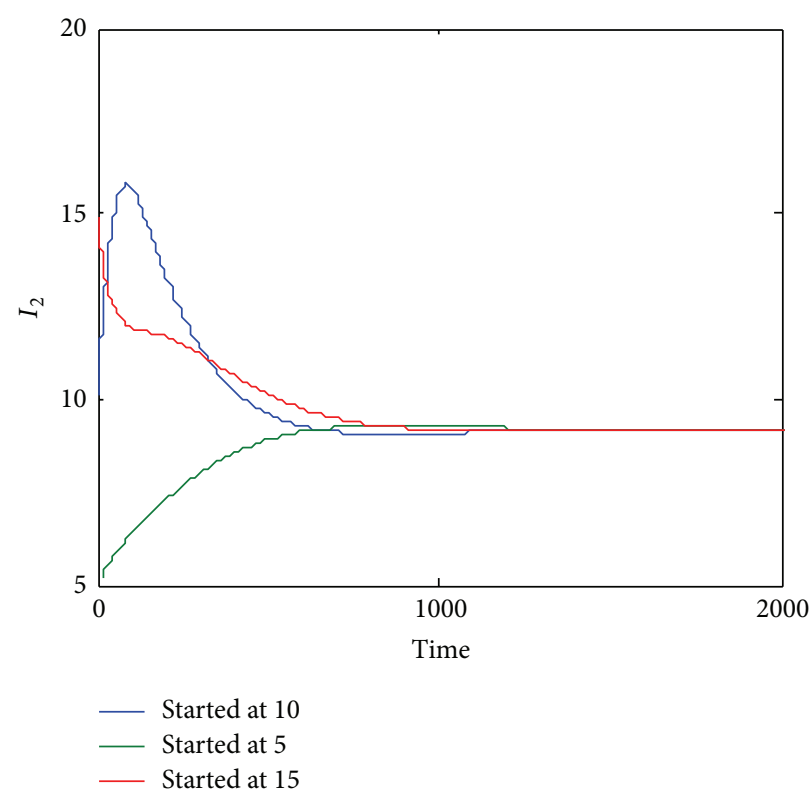

(c)

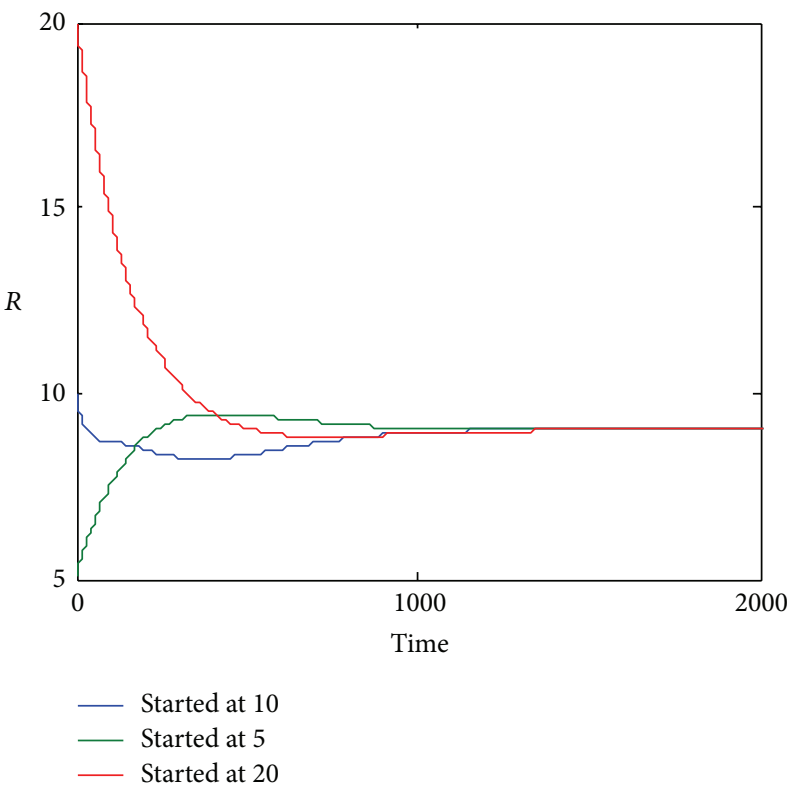

(d)

FIGURE 1: Globally asymptotically stable positive equilibrium point of model (1) for the parameters set (81), started from different sets of initial point.

the solution of model (1) approaches asymptotically to the endemic equilibrium point $E_{3}=(16.4,10.28,9.18,8.99)$ as shown in Figure 1, started from different sets of initial points.

Clearly Figure 1 confirms our obtained analytical results regarding existence of a globally asymptotically stable positive equilibrium point when the parameters values are satisfying $R_{1}>R_{2}>1$. On the other hand, model (1) for the following set of hypothetical data approaches asymptotically to the DFE as shown in Figure 2:

$$
\begin{gathered}
\Lambda=20, \\
\beta_{1}=0.05,
\end{gathered}
$$

$\beta_{2}=0.05$,

$\gamma=0.6$,

$p_{1}=0.01$,

$p_{2}=0.03$,

$\eta=0.5$,

$\mu=0.9$,

$\alpha_{1}=0.1$,

$\alpha_{2}=0.1$,

$\delta=0.3$. 


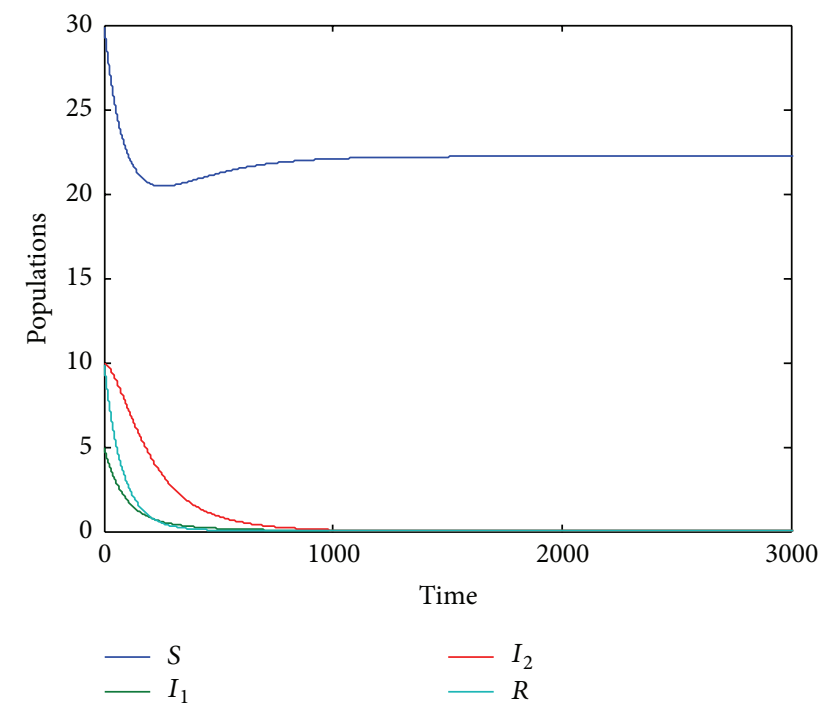

FIgURE 2: Time series of the solution of model (1) that approaches asymptotically to DFE for the data (82).

It is easy to verify that for the data (82) we have $R_{0}=0.86<1$, and the solution approaches to $E_{0}=(22.22,0,0,0)$.

Now in order to investigate the effect of varying one parameter value at a time on the dynamical behavior of model (1), the following results are observed.

(i) Varying of the parameters values $\left(\Lambda, \gamma, p_{2}, \eta, \alpha_{1}, \alpha_{2}, \delta\right)$ does not affect the dynamical behavior of model (1); that is, the system still approaches to coexistence equilibrium point.

(ii) For the data (81) with $\beta_{1} \leq 0.015$, the solution of model (1) approaches asymptotically to $E_{1}=$ $\left(S, 0, I_{2}, 0\right)$ in the interior of positive quadrant of $S I_{2}$ plane with $R_{1}<1<R_{2}$. However for $0.015<$ $\beta_{1}<0.066$ the solution of the model still approaches asymptotically to $E_{1}$ even when $1<R_{1}<R_{2}$. Finally when $\beta_{1} \geq 0.066$ the solution of model (1) approaches to coexistence equilibrium point with $1<R_{2}<R_{1}$ as shown in Figure 3.

(iii) Similar results are obtained in case of varying the parameter $\beta_{2}$ keeping the rest of parameters values in (81) fixed. In fact for $\beta_{2} \leq 0.024$, we have $R_{2}<$ $1<R_{1}$, and the solution of model (1) approaches asymptotically to $E_{2}=\left(S, I_{1}, 0, R\right)$. However for $0.024<\beta_{2}<0.06$, we have $1<R_{2}<R_{1}$, and it is observed that the solution of model (1) approaches asymptotically to $E_{2}=\left(S, I_{1}, 0, R\right)$ too, while when $\beta_{2} \geq 0.06$ the solution of model (1) approaches asymptotically to $E_{3}=\left(S, I_{1}, I_{2}, R\right)$ as shown in Figure 4.

(iv) Now decreasing the parameter $\mu$, keeping the rest of the parameters values in (81) fixed, gives similar dynamical behavior as that of varying $\beta_{2}$. Further it is observed that when $\mu>0.9$, for which $R_{2}<1<R_{1}$, the solution of model (1) approaches asymptotically to $E_{2}=\left(S, I_{1}, 0, R\right)$; however when $0.57<\mu<0.9$, we have $1<R_{2}<R_{1}$, and the solution of model (1) still approaches to $E_{2}=\left(S, I_{1}, 0, R\right)$. Finally, for $\mu \leq 0.57$ that satisfies $1<R_{2}<R_{1}$, the solution of model (1) approaches asymptotically to $E_{3}=$ $\left(S, I_{1}, I_{2}, R\right)$. Clearly this confirmed our obtained existence conditions (10) and (12) as well as stability conditions of these points.

(v) Finally, varying the parameter $p_{1}$, keeping the rest of the parameters values in (81) fixed, showed that, for $p_{1} \geq 0.6$, that satisfies $1<R_{2}<R_{1}$, the solution of model (1) approaches asymptotically to $E_{2}=(S$, $\left.I_{1}, 0, R\right)$; however when $p_{1}<0.6$, which satisfies $1<R_{2}<R_{1}$ too, the solution of model (1) approaches asymptotically to $E_{3}=\left(S, I_{1}, I_{2}, R\right)$ as shown in Figure 5.

\section{Conclusion}

In this paper, we proposed and analyzed an epidemic model involving vertical and horizontal transmission of infection with nonlinear incidence rate. It is assumed that the rates of infections $p_{1}, p_{2}$ are less than the recovery rates $\delta$ and $\gamma$, respectively. According to the diseases in model (1) the population is divided into four subclasses: susceptible individuals that are represented by $S(t)$, infected individuals for SIRS-type of disease that are represented by $I_{1}(t)$, infected individuals for SIS-type of disease that are represented by $I_{2}(t)$, and recovery individuals that are denoted by $R(t)$. The boundedness and invariant of the model are discussed. The basic reproduction number of the model and the associated threshold parameter values, namely, $T_{i}, i=1,2$, are determined. It is observed that if the basic reproduction number is less than unity then the diseases are eradicated from the model. The competitive exclusion principle occurred in model (1) such that only the second disease-free equilibrium point appeared in case of $R_{2}<1<R_{1}$. However only the first disease-free equilibrium point appeared in case of $R_{1}<1<$ $R_{2}$. Finally the coexistence of both the diseases occurred in case of $1<R_{2}<R_{1}$ and the sufficient condition (12) holds. The dynamical behavior of model (1) has been investigated locally as well as globally using Routh-Hurwitz criterion and Lyapunov function, respectively. The local bifurcations of model (1) and the Hopf bifurcation around the endemic equilibrium point are studied. Finally to understand the effect of varying each parameter on the global dynamics of system (1) and to confirm our obtained analytical results, model (1) has been solved numerically and the following results are obtained for the set of hypothetical parameters values given by (81).

(1) Model (1) approaches asymptotically to a globally asymptotically stable point $E_{3}=(16.4,10.28,9.18$, 8.99).

(2) Varying one of the parameters values $\left(\Lambda, \gamma, p_{2}, \eta\right.$, $\left.\alpha_{1}, \alpha_{2}, \delta\right)$ at a time keeping other parameters fixed has no effect on the dynamical behavior of the model.

(3) As the infection rate of the first disease $\left(\beta_{1}\right)$ decreases keeping other parameters fixed as in (81) the solution 


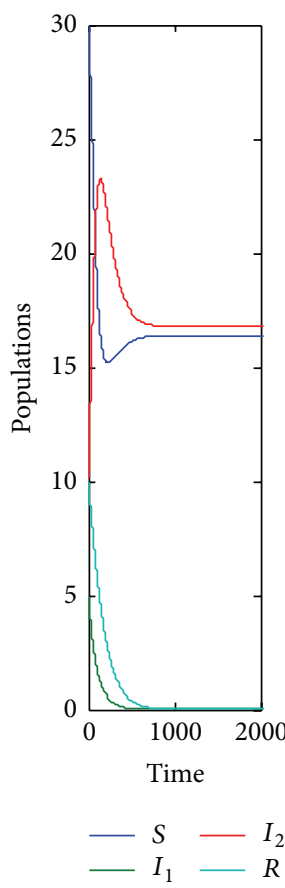

(a)

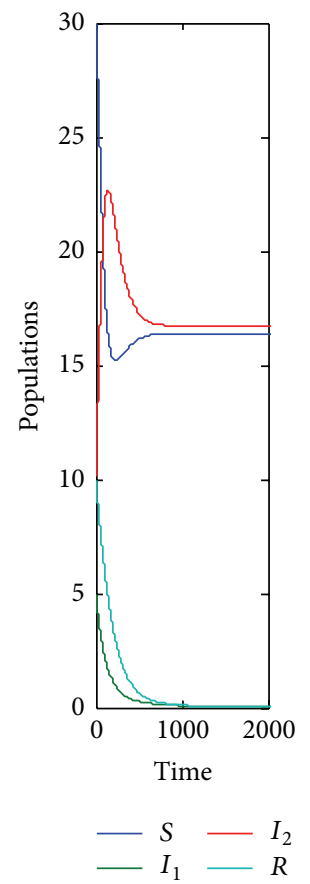

(b)

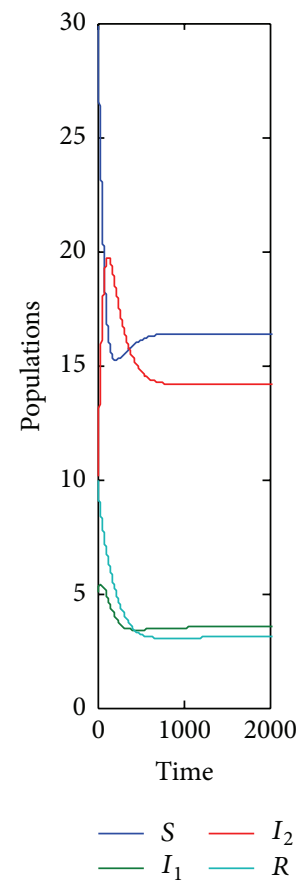

(c)

Figure 3: Time series of the solution of model (1) for the data given by (81). (a) For $\beta_{1}=0.015$ the model approaches to $E_{1}=(16.4,0,16.75,0)$. (b) For $\beta_{1}=0.06$ the model approaches to the same point. (c) For $\beta_{1}=0.3$ the model approaches to $E_{3}=(16.4,3.51,14.1,3.07)$.

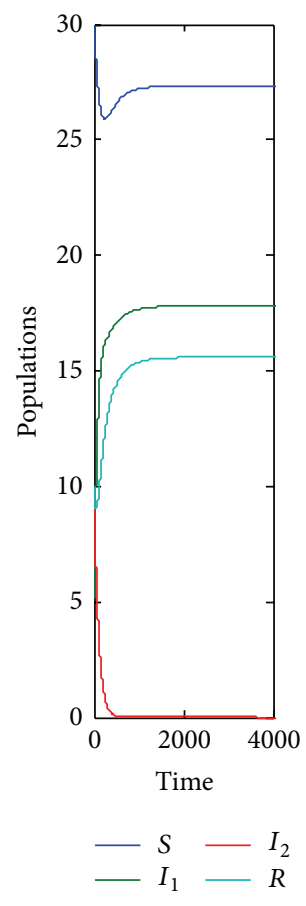

(a)

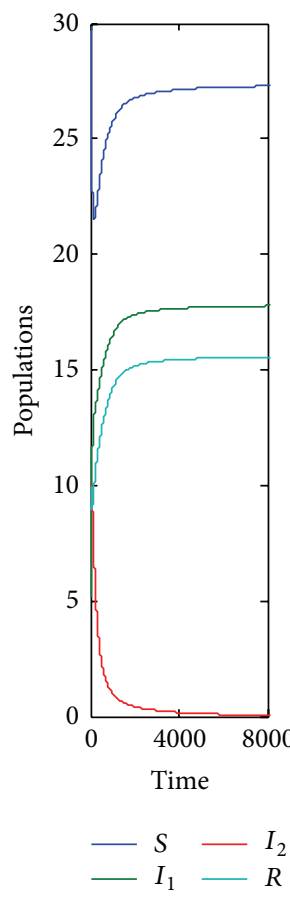

(b)

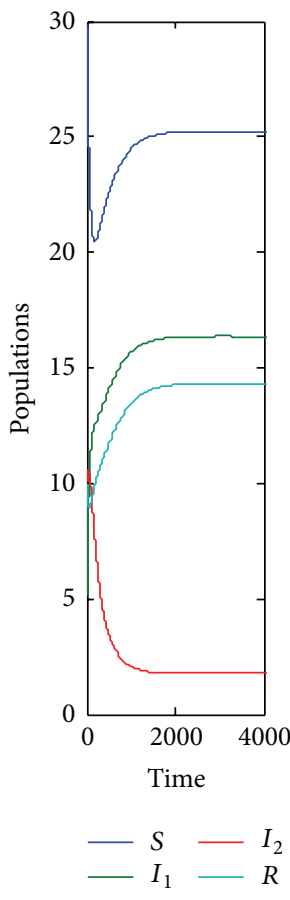

(c)

Figure 4: Time series of the solution of model (1) for the data given by (81). (a) For $\beta_{2}=0.024$ the model approaches to $E_{2}=$ $(27.33,17.80,0,15.5)$. (b) For $\beta_{2}=0.059$ the model approaches to the same point. (c) For $\beta_{2}=0.065$ the model approaches to $E_{3}=$ $(25.2,16.3,1.76,14.3)$. 


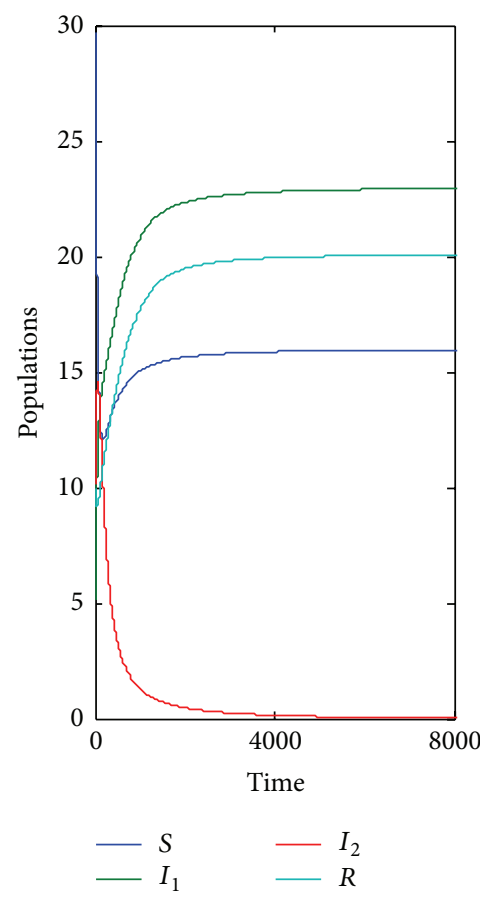

(a)

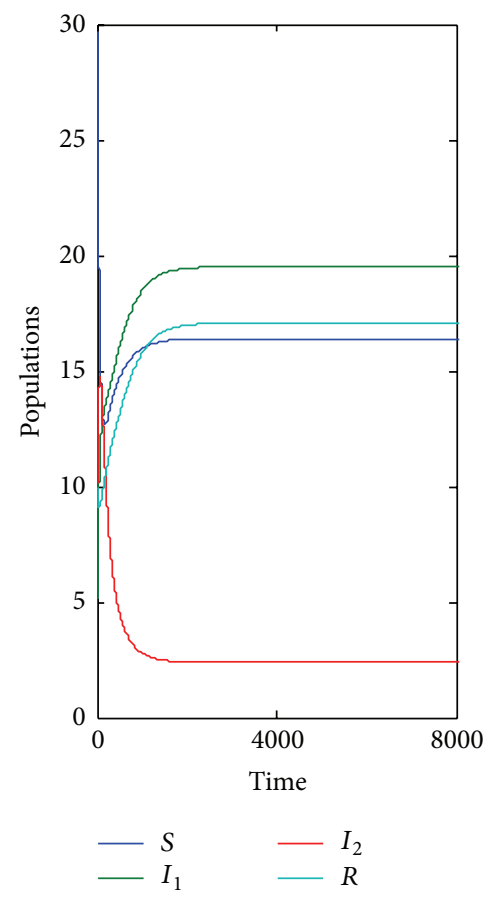

(b)

Figure 5: Time series of the solution of model (1) for the data given by (81). (a) For $p_{1}=0.6$ the model approaches to $E_{2}=(15.9,22.9,0,20.08)$.

(b) For $p_{1}=0.5$ the model approaches to $E_{3}=(16.4,19.5,2.40,17.06)$.

of model (1) approaches asymptotically to the equilibrium point $E_{1}=(16.4,0,16.75,0)$. However in case of increasing this parameter the model is still globally asymptotically stable in the interior of $\mathbb{R}_{+}^{4}$.

(4) As the infection rate of the second disease $\left(\beta_{2}\right)$ decreases keeping other parameters fixed as in (81) the solution of model (1) approaches asymptotically to the equilibrium point $E_{2}=(27.33,17.80,0,15.5)$. However in case of increasing this parameter the model is still globally asymptotically stable in the interior of $\mathbb{R}_{+}^{4}$.

(5) As the mortality rate $(\mu)$ increases keeping other parameters fixed as in (81) the solution of model (1) approaches asymptotically to the equilibrium point $E_{2}=(13.8,5.16,0,2.5)$ and when $\mu$ decreases the model is still globally asymptotically stable in the interior of $\mathbb{R}_{+}^{4}$. Further, it is observed that $p_{1}$ has the same effect as $\mu$ on the dynamical behavior of model (1).

(6) For the parameter set given in (82) the solution of model (1) approaches asymptotically to DFE.

(7) Finally, although for our selected parameters values model (1) does not undergo periodic dynamics, the model still has possibility to have periodic dynamics for other sets of parameters, especially Hopf bifurcation existing analytically.

\section{Conflict of Interests}

The authors declare that there is no conflict of interests regarding the publication of this paper.

\section{References}

[1] G. Hardin, “The competitive exclusion principle," Science, vol. 131, no. 3409, pp. 1292-1297, 1960.

[2] A. Iggidr, J.-C. Kamgang, G. Sallet, and J.-J. Tewa, "Global analysis of new malaria intrahost models with a competitive exclusion principle," SIAM Journal on Applied Mathematics, vol. 67, no. 1, pp. 260-278, 2006.

[3] V. Volterra, "Variations and fluctuations of the number of individuals in animal species living together," Journal du Conseil International pour l'Exploration de la Mer, vol. 3, no. 1, pp. 3-51, 1928.

[4] A. S. Ackleh and L. J. Allen, "Competitive exclusion in SIS and SIR epidemic models with total cross immunity and densitydependent host mortality," Discrete and Continuous Dynamical Systems-Series B, vol. 5, no. 2, pp. 175-188, 2005.

[5] F. Brauer, J. Wu, and P. van den Driessche, Mathematical Epidemiology, Springer, Berlin, Germany, 2008.

[6] P. van den Driessche and J. Watmough, "Reproduction numbers and sub-threshold endemic equilibria for compartmental models of disease transmission," Mathematical Biosciences, vol. 180, no. 1-2, pp. 29-48, 2002.

[7] J. M. Heffernan, R. J. Smith, and L. M. Wahl, "Perspectives on the basic reproductive ratio," Journal of the Royal Society Interface, vol. 2, no. 4, pp. 281-293, 2005. 
[8] O. Diekmann, J. A. P. Heesterbeek, and J. A. J. Metz, "On the definition and the computation of the basic reproduction ratio $R_{o}$ in models for infectious diseases in heterogeneous populations in models for infectious diseases in heterogeneous populations," Journal of Mathematical Biology, vol. 28, no. 4, pp. 365-382, 1990.

[9] M. Martcheva, "A non-autonomous multi-strain SIS epidemic model," Journal of Biological Dynamics, vol. 3, no. 2-3, pp. 235251, 2009.

[10] A. S. Ackleh and L. J. S. Allen, "Competitive exclusion and coexistence for pathogens in an epidemic model with variable population size," Journal of Mathematical Biology, vol. 47, no. 2, pp. 153-168, 2003.

[11] M. Lipsitch, S. Siller, and M. A. Nowak, "The evolution of virulence in pathogens with vertical and horizontal transmission," Evolution, vol. 50, no. 5, pp. 1729-1741, 1996.

[12] D. Bichara, A. Iggidr, and G. Sallet, "Global analysis of multistrains SIS, SIR and MSIR epidemic models," Journal of Applied Mathematics and Computing, vol. 44, no. 1-2, pp. 273-292, 2014.

[13] M. Y. Li, H. L. Smith, and L. Wang, "Global dynamics an SEIR epidemic model with vertical transmission," SIAM Journal on Applied Mathematics, vol. 62, no. 1, pp. 58-69, 2001.

[14] W. M. Liu, H. W. Hethcote, and S. A. Levin, "Dynamical behavior of epidemiological models with nonlinear incidence rates," Journal of Mathematical Biology, vol. 25, no. 4, pp. 359380, 1987.

[15] O. Adebimpe, A. A. Waheed, and B. Gbadamosi, "Modeling and analysis of an SEIRS epidemic model with saturated incidence," Journal of Engineering Research and Application, vol. 3, no. 5, pp. 1111-1116, 2013.

[16] R. K. Naji and A. N. Mustafa, "The dynamics of an ecoepidemiological model with nonlinear incidence rate," Journal of Applied Mathematics, vol. 2012, Article ID 852631, 24 pages, 2012.

[17] C. V. De-León, "On the global stability of SIS, SIR and SIRS epidemic models with standard incidence," Chaos, Solitons \& Fractals, vol. 44, no. 12, pp. 1106-1110, 2011.

[18] J. P. LaSalle, "Stability theory for ordinary differential equations," Journal of Differential Equations, vol. 4, no. 1, pp. 57-65, 1968.

[19] E. Shim, An epidemic model with immigration of infectives and vaccination [M.S. thesis], Department of Mathematics, Institute of Applied Mathematics, University of British Columbia, Vancouver, Canada, 2004.

[20] L. Perko, Differential Equation and Dynamical Systems, Springer, New York, NY, USA, 3rd edition, 2001.

[21] M. M. A. El-Sheikh and S. A. A. El-Marouf, "On stability and bifurcation of solutions of an SEIR epidemic model with vertical transmission," International Journal of Mathematics and Mathematical Sciences, vol. 2004, no. 56, pp. 2971-2987, 2004.

[22] X. Zhou and J. Cui, "Analysis of stability and bifurcation for an SEIV epidemic model with vaccination and nonlinear incidence rate," Nonlinear Dynamics, vol. 63, no. 4, pp. 639-653, 2011. 


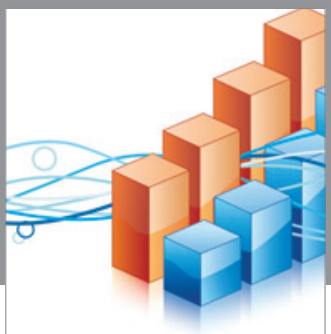

Advances in

Operations Research

vatem alat4

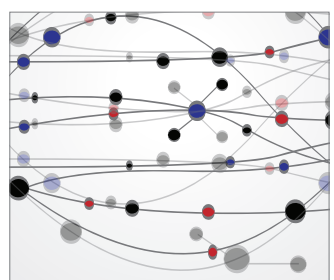

\section{The Scientific} World Journal
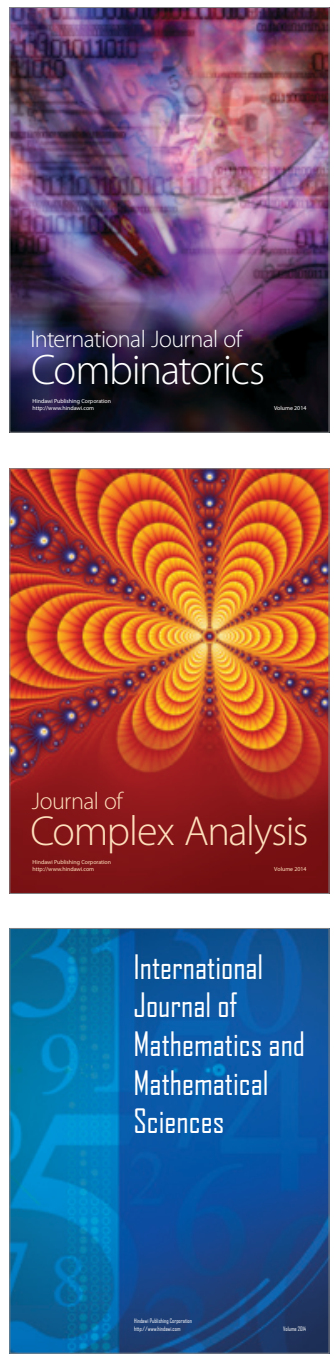
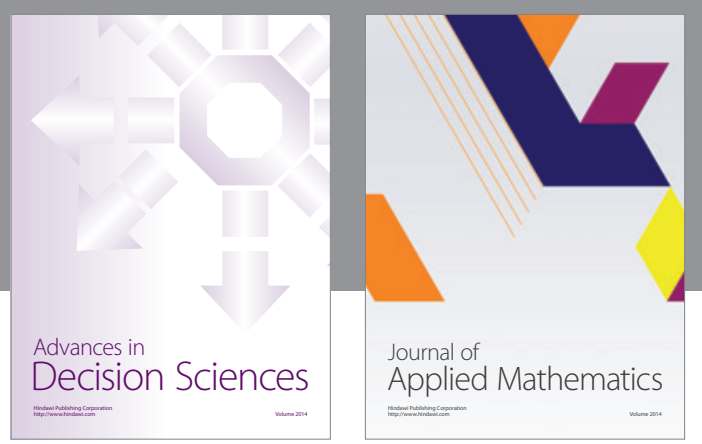

Algebra

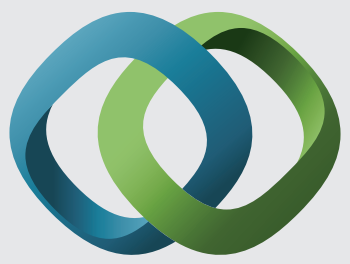

\section{Hindawi}

Submit your manuscripts at

http://www.hindawi.com
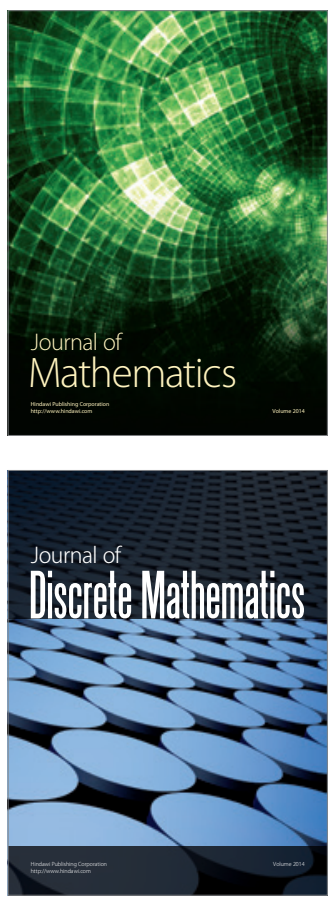

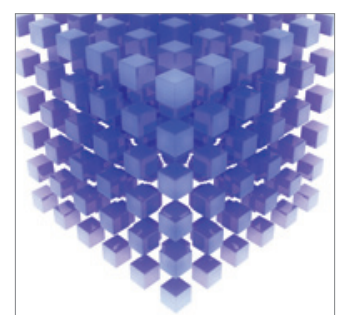

Mathematical Problems in Engineering
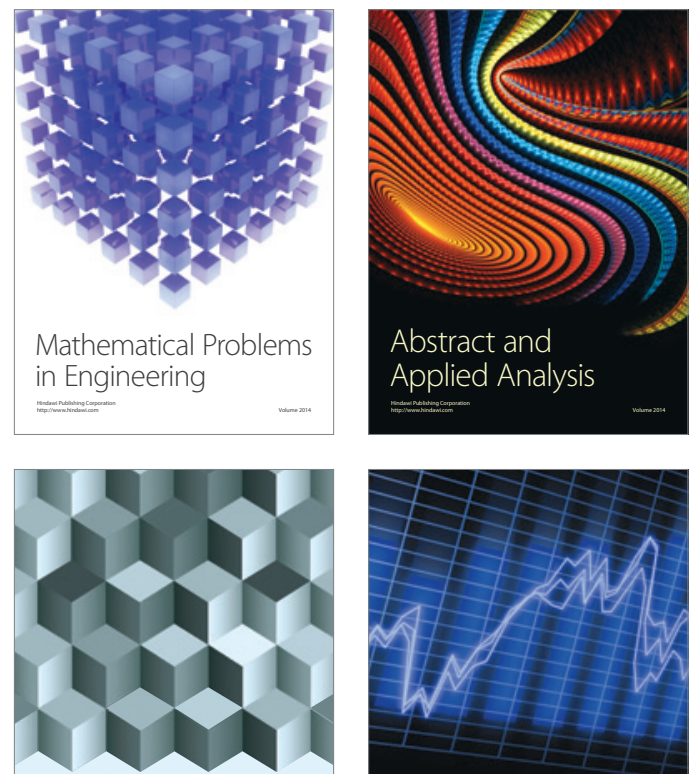

Journal of

Function Spaces

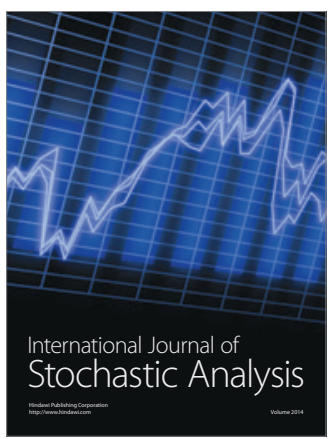

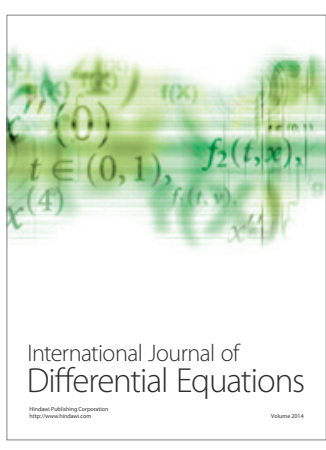
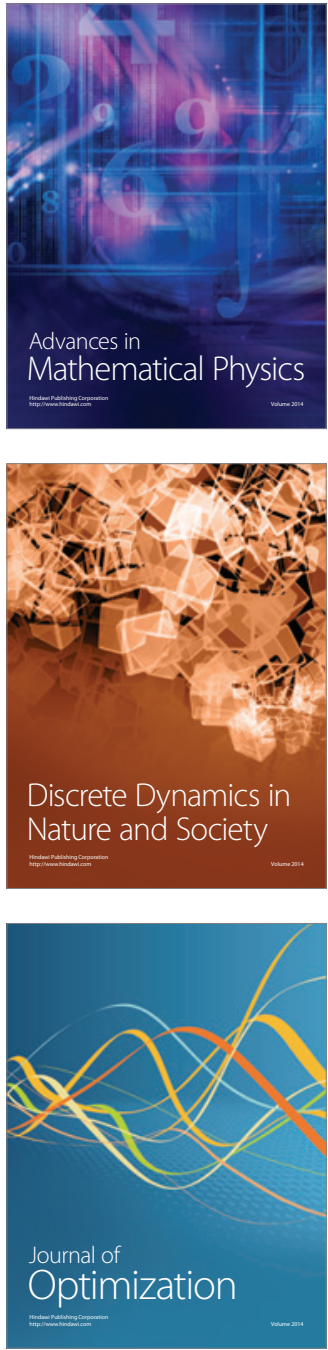